\title{
Supplementary Information: Revisiting the classic activity coefficient models
}

Walter W. Focke, ${ }^{1, *}$ Stefan Endres, ${ }^{1}$ Elizabeth L. du Toit ${ }^{1}$ and Mattheüs T. Loots ${ }^{1}$ and Roelof L. J. Coetzer ${ }^{2,3}$

${ }^{1}$ Institute of Applied Materials, Department of Chemical Engineering, University of Pretoria, Private Bag X20, Hatfield 0028, Pretoria, South Africa Tel: +27833266549 e-mail: walter.focke@up.ac.za

${ }^{2}$ Data Science, Sasol Operations Improvement, PO Box 1, Sasolburg 1947, South Africa

${ }^{3}$ School of Industrial Engineering, North-West University, Private Bag X6001, Potchefstroom 2520, South Africae-mail: roelof.coetzer@sasol.com

\section{S1. Weighted means ${ }^{1}$}

The following notation is used: $\mathbf{R}_{+}$denotes the set of all positive real numbers and $\mathbf{R}_{+}^{n}$ its $n$-product. Let $a_{1}, a_{1}, \ldots a_{n} \in R_{+}$. The objective is to find a way to represent the vector $\boldsymbol{a}$ by a scalar meaningful quantity $a$ subject to scaling by a vector $\boldsymbol{x} \in R_{+}{ }^{n}$ of normalized weights that are subject to the restrictions $0 \leq x_{i} \leq 1$ and $\sum x_{i}=1$. The weighted power mean is defined as follows:

$$
P_{p}(\mathbf{u}, \boldsymbol{x}, p)=\lim _{q \rightarrow p^{+}}\left[\sum_{i=1}^{n} x_{i} u_{i}^{q}\right]^{1 / q}, p \in \mathbf{R}
$$

This precise definition also admits the special case with $p=0$. Specific cases of power means are presented in Table S1.

Table S1: Special weighted power means

\begin{tabular}{ccc}
\hline Weighted mean & $\boldsymbol{p}$ & Definition \\
\hline Harmonic & -1 & $H=P_{-1}=\left[\sum_{i=1}^{n} \frac{x_{i}}{a_{i}}\right]^{-1}$ \\
Geometric & 0 & $G=P_{0}=\prod_{i=1}^{n} a_{i}^{x_{i}}$ \\
Arithmetic & 1 & $A=P_{1}=\sum_{i=1}^{n} x_{i} a_{i}$ \\
Quadratic & 2 & $Q=P_{2}=\sqrt{\sum x_{i} a_{i}^{2}}$ \\
Power & $p$ & $P_{p}=\left[\sum_{i=1}^{n} x_{i} a_{i}\right]^{1 / p}$ \\
\hline
\end{tabular}

\section{S2. DWPM model development ${ }^{2}$}

Let $\mathbf{R}_{+}$denote the set of all positive real numbers and $\mathbf{R}_{+}^{n}$ its $n$-product. The empirical model $y=y(\boldsymbol{x}, \boldsymbol{A})$ links the physical property of interest with mixture composition. Here $y \in \mathbf{R}_{+}$is the response variable; $\boldsymbol{x} \in \mathbf{R}_{+}^{n}$ is a vector of normalized weights denoting mole fractions subject to the restrictions: $0 \leq x_{i} \leq 1$ and $x_{1}+x_{2}+\ldots .+x_{n}=1$. It is further assumed that the elements $a_{i j} \in \mathbf{R}_{+}$of the coefficient matrix, $\boldsymbol{A}$, fully parameterize the range of possible response behaviours. 
Consider a mixture composed of $n$ different molecular species. The following notation is used: $R_{+}$ denotes the set of all positive real numbers and $R_{+}{ }^{n}$ its $n$-product. Mixture composition is quantified by the vector $z \in R_{+}{ }^{n}$ of normalized weights representing some measure of the concentration of the species in the mixture. These normalized weights are subject to the restrictions $0 \leq z_{i} \leq 1$ and $\Sigma z_{i}=$ 1.

The development of the DWPM mixture model was inspired by Scott's ${ }^{3}$ two-fluid theory. The hypothesis is that a real mixture is composed of imaginary reference fluids defined by the nature of the molecule at the centre of a fluid cluster (See Figure 1). The property values are expressed in terms of weighted compositions, i.e. mole fractions. In the DWPM, the reference fluid properties, $u_{i}$, are specified by weighted power means of order $s_{i}$ over the $a_{i j}$ :

$$
u_{i}(\boldsymbol{x})=\lim _{p \rightarrow s_{i}^{+}}\left(\sum_{k=1}^{n} x_{k} a_{i k}^{p}\right)^{1 / p}
$$

The overall mixture property $a$ is also specified by a power mean of order $r$ over the reference fluids

$$
a=\lim _{p \rightarrow r^{+}}\left(\sum_{i=1}^{n} x_{i}\left[u_{i}(\mathbf{x})\right]^{p}\right)^{1 / p}=\lim _{p \rightarrow r^{+}}\left(\sum_{i=1}^{n} x_{i}\left[\lim _{q \rightarrow s_{i}^{+}}\left(\sum_{k=1}^{n} x_{k} a_{i k}^{q}\right)^{1 / q}\right]^{p}\right)^{1 / p}
$$

Note, that in its most elaborate form, the $s_{i}$ values are allowed to be different for each reference fluid. The simplest model assumes that all the $s_{i}$ values are equal unity. The values are determined by the nature of the central molecule of the liquid cell. However, a single value of the exponent $r$ is used to average over the assembly of cells.

The DWPM model can be understood in terms of Scott's ${ }^{3}$ two-fluid theory. Equation (S-2) specifies the procedure for calculating the properties of the reference fluids. Equation (S-3) defines how these are to be combined to determine the final mixture property.

The generalized weighted power mean defined by Equation (S-3) is homogeneous of order one in the coefficients $a_{i j}$. It was shown that this implies that the model is well-conditioned with respect to the adjustable parameters. ${ }^{4,5}$ 


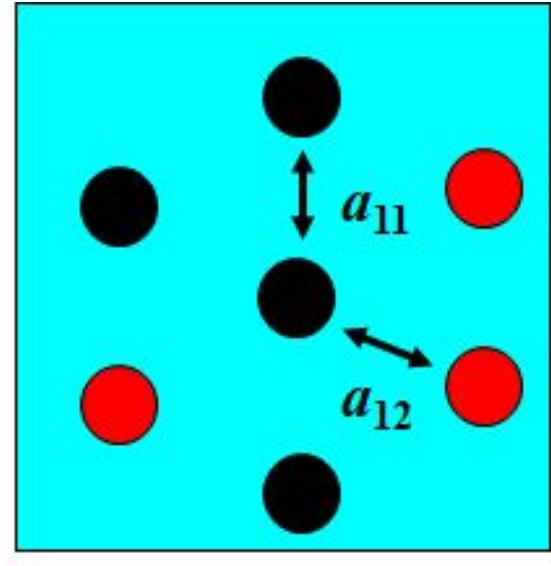

Hypothetical fluid (1)

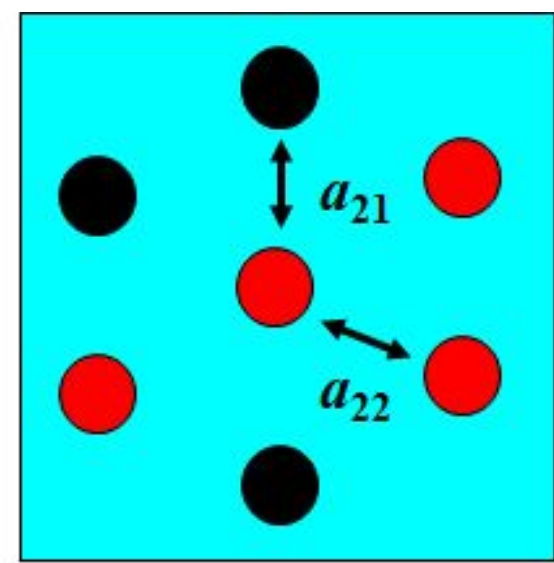

Molecule of component 1

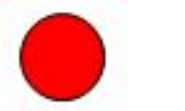

Molecule of component 2

\section{Hypothetical fluid (2)}

Figure S-1: Schematic illustration of Scotts's ${ }^{3}$ two-fluid theory for a binary mixture. In cell (1) a molecule of component (1) is located at the centre whereas, cell (2) has a molecule of component (2) at the centre.

\section{S3. The Michelsen-Kistenmacher syndrome}

Michelsen and Kistenmacher ${ }^{6}$ stated that one of the requirements of a consistent mixing rule is that it must be invariant with respect to dividing one component into two or more identical subcomponents. This also applies to expressions for the excess Gibbs energy. ${ }^{6}$ To be fair, one should start with the hypothesis that the model under consideration does in fact comply with the required consistency requirement. To prove that this is true is often very difficult to do. However, in order to show that it is not the case, it is sufficient to show a counter example even if this is done for a simplified version of the model, e.g. for a ternary mixture. This means that the analysis can be limited to binary and ternary systems in the event that the model can be shown to fail the specified consistency requirement.

Whether a model conforms to the invariance principle can be tested in two different ways. The first approach starts with the ternary model and it is then assumed that two of the components were actually the same compound. Mixing portions of the same compound results in an ideal solution so the contribution to the excess Gibbs energy will be zero. This is taken into account and the model is examined to determine whether it reduces to the binary form. The second method starts with the binary model form, e.g. component 1 mixed with component 4 . Component 4 is then divided into two new ones, components 2 and 3 (with $x_{4}=x_{2}+x_{3}$ ) in order to generate a pseudo ternary mixture. The check is to see whether the proposed ternary expression can be constructed in this way.

\section{S3.1. Margules multicomponent ${ }^{7}$}

Oliver ${ }^{7}$ reviewed the different multicomponent forms suggested for the Margules model. He showed that, for a ternary mixture, the activity coefficients can all be reduced to the following common form: 


$$
\begin{aligned}
\operatorname{In} \gamma_{i} & =A_{i j}\left[x_{j}^{2}+0.5 x_{j} x_{k}-2 x_{i} x_{j}^{2}-x_{i} x_{j} x_{k}\right]+A_{j i}\left[0.5 x_{j} x_{k}+2 x_{i} x_{j}^{2}+x_{i} x_{j} x_{k}\right] \\
& +A_{i k}\left[x_{k}^{2}+0.5 x_{j} x_{k}-2 x_{i} x_{k}^{2}-x_{i} x_{j} x_{k}\right]+A_{k i}\left[0.5 x_{j} x_{k}+2 x_{i} x_{k}^{2}+x_{i} x_{j} x_{k}\right] \\
& +A_{j k}\left[0.5 x_{j} x_{k}-2 x_{j} x_{k}^{2}-x_{i} x_{j} x_{k}\right]+A_{k j}\left[-1.5 x_{j} x_{k}+2 x_{j} x_{k}^{2}+x_{i} x_{j} x_{k}\right]
\end{aligned}
$$

Algebraic manipulation leads to the following expression for the excess Gibbs energy:

$$
\begin{aligned}
& g^{E}\left(x_{i}, x_{j}, x_{k}\right)=x_{i} \ln \gamma_{i}+x_{j} \ln \gamma_{j}+x_{k} \ln \gamma_{k} \\
& =x_{i} x_{j}\left[A_{i j}\left(0.5 x_{k}+x_{j}\right)+A_{j i}\left(0.5 x_{k}+x_{i}\right)\right] \\
& +x_{i} x_{k}\left[A_{i k}\left(0.5 x_{j}+x_{k}\right)+A_{k i}\left(0.5 x_{j}+x_{i}\right)\right] \\
& +x_{j} x_{k}\left[A_{j k}\left(0.5 x_{i}+x_{k}\right)+A_{k j}\left[0.5 x_{i}+x_{j}\right]\right]
\end{aligned}
$$

Setting one of the mole factions equal to zero, e.g. $x_{k}=0$, recovers the conventional expression for the binary Margules form:

$$
g^{E}=x_{i} \ln \gamma_{i}+x_{j} \ln \gamma_{j}=x_{i} x_{j}\left[A_{i j} x_{j}+A_{j i} x_{i}\right]
$$

Starting from the ternary expressions, consider what happens when components $j$ and $k$ are actually the same compound. Let that compound be denoted by component $m$. This means the ternary mixture is in fact a binary mixture of component $i$ with component $m$. This means that $x_{m}=x_{j}+x_{k}$. Since mixing portions of pure components yield an ideal solution, it follows that $A_{j k}, A_{k j}=0$. Furthermore, it also holds that $A_{i j}=A_{i k}=A_{i m}$ and $A_{j i}=A_{k i}=A_{m, i}$. Substituting and simplifying results in

$$
g^{E}\left(x_{i}, x_{m}=x_{j}+x_{k}\right)=x_{i}\left[A_{i m}\left\{x_{j} x_{j}+x_{j} x_{k}+x_{k} x_{k}\right\}+A_{m i}\left\{x_{i} x_{j}+x_{j} x_{k}+x_{i} x_{k}\right\}\right]
$$

In the second approach, start with the binary form

$$
g^{E}=x_{i} x_{m}\left[A_{i m} x_{m}+A_{m i} x_{i}\right]
$$

Now substitute $x_{m}=x_{j}+x_{k}$ :

$$
g^{E}\left(x_{i}, x_{m}=x_{j}+x_{k}\right)=x_{i}\left[A_{i m}\left(x_{j}^{2}+2 x_{j} x_{k}+x_{k}^{2}\right)+A_{m i}\left(x_{i} x_{j}+x_{i} x_{k}\right)\right]
$$

These two equations for $g^{E}\left(x_{i}, x_{m}=x_{j}+x_{k}\right)$ are not equivalent. This means that the proposed ternary model is not invariant with respect to dividing one of the components into two identical components. The implication is that it suffers from the Michelsen-Kistenmacher syndrome. ${ }^{6}$

\section{S3.2. CALPHAD Redlich-Kister multicomponent excess Gibbs energy $\operatorname{model}^{8,9}, 10$}

CALPHAD implements Redlich-Kister expansions using the Muggianu $^{8}$ formalism ${ }^{10}$. The equation describing a ternary system is:

$$
g^{E}=x_{1} x_{2} \sum_{i=o}^{i=n_{12}} L_{12, i}\left(x_{1}-x_{2}\right)^{i}+x_{1} x_{3} \sum_{j=o}^{j=n_{13}} L_{12, j}\left(x_{1}-x_{3}\right)^{j}+x_{2} x_{3} \sum_{i=o}^{i=n_{12}} L_{23, k}\left(x_{2}-x_{3}\right)^{k}
$$

Here a slightly different notation will be used and the analysis will be limited to binary and ternary mixtures described by a truncated expression in which only the first two terms in the expansion are kept. The expression for a binary mixture is:

$$
g^{E}\left(x_{1}, x_{2}\right)=A_{12} x_{1} x_{2}+B_{12} x_{1} x_{2}\left(x_{1}-x_{2}\right)
$$

The ternary system will be represented by: 


$$
g^{E}\left(x_{1}, x_{2}, x_{3}\right)=A_{12} x_{1} x_{2}+B_{12} x_{1} x_{2}\left(x_{1}-x_{2}\right)+A_{13} x_{1} x_{3}+B_{13} x_{1} x_{3}\left(x_{1}-x_{3}\right)+A_{23} x_{2} x_{3}+B_{23} x_{2} x_{3}\left(x_{2}-x_{3}\right)
$$

Now consider the possibility of telescoping the ternary into a binary expression by postulating that components 2 and 3 were actually the same. Relabel that component as component 4 . This means that $x_{4}=x_{2}+x_{3}$. Since mixing portions of pure component results in an ideal solution, it follows that $A_{23}, B_{32}=0$. Furthermore, it is also true that $A_{12}=A_{13}=A_{14}$ and $B_{12}=B_{13}=B_{14}$. Substituting, yields after simplification:

$$
g^{E}\left(x_{1}, x_{4}=x_{2}+x_{3}\right)=A_{14} x_{1} x_{4}+B_{14} x_{1} x_{4}\left[x_{1}-x_{4}-2 x_{1} x_{2} / x_{4}\right]
$$

This equation would agree with the expected form only if the last term in the square brackets were absent. Therefore, it can be said that the ternary model suffers from the Michelsen-Kistenmacher syndrome. ${ }^{6}$

\section{S3.3. Multicomponent van Laar expression ${ }^{11}$}

The following expressions were proposed for a multicomponent extension of the van Laar activity coefficient model: ${ }^{11}$

$$
\ln \gamma_{i}=A_{i}\left(1-z_{i}\right)^{2}\left[1+2 z_{i}\left(\frac{A_{i} B_{i}}{\left|A_{i} B_{i}\right|}-1\right)\right]
$$

where $z_{i}=\frac{\left|A_{i}\right| x_{i}}{\left|A_{i}\right| x_{i}+\left|B_{i}\right|\left(1-x_{i}\right)} ; A_{i}=\frac{1}{\left(1-x_{i}\right)} \sum_{j=1}^{3} x_{j} A_{i j} ; \quad B_{i}=\frac{1}{\left(1-x_{i}\right)} \sum_{j=1}^{3} x_{j} A_{j i}$

The use of absolute values presumably caters for the effects of negative coefficients. For simplicity, and to avoid the issues associated with the absolute values, the present analysis will consider ternary systems for which all the coefficients are positive, i.e. $A_{i}, B_{i}>0 \forall i$. These assumptions result in significant simplifications:

$$
\begin{aligned}
& \text { In } \gamma_{i}=\left(1-x_{i}\right)\left[x_{j} A_{i j}+x_{k} A_{i k}\right]\left[\frac{x_{j} A_{j i}+x_{k} A_{k i}}{\left(x_{j} A_{i j}+x_{k} A_{i k}\right) x_{i}+\left(x_{j} A_{j i}+x_{k} A_{k i}\right)\left(1-x_{i}\right)}\right]^{2} \\
& g^{E}=x_{1} \ln \gamma_{1}+x_{2} \operatorname{I} n \gamma_{2}+x_{3} \operatorname{In} \gamma_{3} \\
& \quad=\frac{x_{1}\left(1-x_{1}\right)\left(x_{2} A_{12}+x_{3} A_{13}\right)\left(x_{2} A_{21}+x_{3} A_{31}\right)^{2}}{\left[\left(x_{2} A_{12}+x_{3} A_{13}\right) x_{1}+\left(x_{2} A_{21}+x_{3} A_{31}\right)\left(1-x_{1}\right)\right]^{2}} \\
& \quad+\frac{x_{2}\left(1-x_{2}\right)\left(x_{1} A_{21}+x_{3} A_{23}\right)\left(x_{1} A_{12}+x_{3} A_{32}\right)^{2}}{\left[\left(x_{1} A_{21}+x_{3} A_{23}\right) x_{2}+\left(x_{1} A_{12}+x_{3} A_{32}\right)\left(1-x_{2}\right)\right]^{2}} \\
& +\frac{x_{3}\left(1-x_{3}\right)\left(x_{1} A_{31}+x_{2} A_{32}\right)\left(x_{1} A_{13}+x_{2} A_{23}\right)^{2}}{\left[\left(x_{1} A_{31}+x_{2} A_{32}\right) x_{3}+\left(x_{1} A_{13}+x_{2} A_{23}\right)\left(1-x_{3}\right)\right]^{2}}
\end{aligned}
$$

Consider the possibility that two of the components (e.g. components 2 and 3 ) in the ternary mixture were actually identical. In that case, one has a binary mixture for which the van Laar expression is:

$$
g^{E}=\frac{A_{41} A_{14} x_{1} x_{4}}{x_{1} A_{14}+x_{4} A_{41}}
$$


Let the common compound be component 4 . This means that that $x_{4}=x_{2}+x_{3}$. Since mixing portions of pure component results in an ideal solution, it follows that $A_{23}, A_{32}=0$. Furthermore, $A_{12}=A_{13}=$ $A_{14} ; A_{21}=A_{31}=A_{41}$. Substitute these expressions into the equation describing the ternary system and subtract the equation describing the binary mixture. This should yield a result exactly equal to zero. However, after simplification the result is:

$$
x_{1} A_{41} A_{14}^{2}\left\{\frac{x_{2}\left(1-x_{2}\right)}{\left[x_{2} A_{41}+\left(1-x_{2}\right) A_{14}\right]^{2}}+\frac{x_{3}\left(1-x_{3}\right)}{\left[x_{3} A_{41}+\left(1-x_{3}\right) A_{14}\right]^{2}}-\frac{x_{1} x_{4}}{\left[x_{1} A_{14}+\left(1-x_{1}\right) A_{41}\right]^{2}}\right\} \neq 0
$$

This indicates that the proposed multicomponent expression suffers from the Michelsen-

Kistenmacher syndrome. ${ }^{6}$

\section{S4. The invariance of the excess Gibbs energy to linear composition shifts in the Gibbs energy}

This Section will show that shifting $g^{\#}$ by a linear blending rule expression in the mole fractions does not affect the resulting $g^{\mathrm{E}}$. This will then be used to show that the van Laar-type expressions of Table 1 in the main text are fundamentally equivalent expressions. Consider the effect of a linear shift in $g^{\#}$ in order to generate a new Gibbs function $f^{\# \text { : }}$

$f^{\#}=g^{\#}+d_{1} x_{1}+d_{2} x_{2}$

Let:

$g_{1}^{\#}=g^{\#}\left(x_{1}=1\right)$ and $g_{2}^{\#}=g^{\#}\left(x_{2}=1\right)$

$f_{1}^{\#}=f^{\#}\left(x_{1}=1\right)=g_{1}^{\#}+d_{1}$ and $f_{2}^{\#}=f^{\#}\left(x_{2}=1\right)=g_{2}^{\#}+d_{2}$

Now:

$$
\begin{aligned}
f^{E} & =f^{\#}-x_{1} f_{1}^{\#}-x_{2} f_{2}^{\#} \\
& =g^{\#}+d_{1} x_{1}+d_{2} x_{2}-x_{1}\left(g_{1}^{\#}+d\right)-x_{2}\left(g_{2}^{\#}+d_{2}\right) \\
& =g^{\#}-x_{1} g_{1}^{\#}-x_{2} g_{2}^{\#} \\
& =g^{E}
\end{aligned}
$$

This proves that the excess Gibbs energy $g^{\mathrm{E}}$ is invariant with respect of a linear composition shift in the Gibbs free energy itself. Clearly this is a "good" thing as one can only measure differences in the Gibbs free energy!

The simplest Padè-based mixture model that generates the van Laar expression is $P_{\mathrm{q}}(0,1)$. For a binary mixture it reads:

$g^{\#}=\frac{a_{o}}{b_{1} x_{1}+b_{2} x_{2}}$

Introduce a linear shift as follows:

$f^{\#}=\frac{a_{o}}{b_{1} x_{1}+b_{2} x_{2}}+d_{1} x_{1}+d_{2} x_{2}=\frac{\left(a_{o}+d_{1} b_{1}\right) x_{1}^{2}+\left(2 a_{o}+d_{2} b_{1}+d_{1} b_{2}\right) x_{1} x_{2}+\left(a_{o}+d_{2} b_{2}\right) x_{2}^{2}}{b_{1} x_{1}+b_{2} x_{2}}$

This corresponds to the Padè $P_{2}(2,2)$ form. 


$$
g^{\#}=\frac{a_{o}}{b_{1} x_{1}+b_{2} x_{2}}
$$

Let:

$$
\begin{aligned}
f^{\#} & =\frac{a_{o}}{b_{1} x_{1}+b_{2} x_{2}}+d_{1} x_{1}+d_{2} x_{2} \\
& =\frac{a_{o}\left(x_{1}+x_{2}\right)^{2}+\left(b_{1} x_{1}+b_{2} x_{2}\right)\left(d_{1} x_{1}+d_{2} x_{2}\right)}{b_{1} x_{1}+b_{2} x_{2}} \\
& =\frac{a_{o}\left(x_{1}^{2}+2 x_{1} x_{2}+x_{2}^{2}\right)+d_{1} b_{1} x_{1}^{2}+d_{2} b_{1} x_{1} x_{2}+\left(d_{1} b_{2} x_{1} x_{2}+d_{2} b_{2} x_{2}^{2}\right)}{b_{1} x_{1}+b_{2} x_{2}} \\
& =\frac{\left(a_{o}+d_{1} b_{1}\right) x_{1}^{2}+\left(2 a_{o}+d_{2} b_{1}+d_{1} b_{2}\right) x_{1} x_{2}+\left(a_{o}+d_{2} b_{2}\right) x_{2}^{2}}{b_{1} x_{1}+b_{2} x_{2}}
\end{aligned}
$$

The numerator reduces to the linear relationship found in the numerator of the Padè $P_{2}(1,1)$ form if $d_{2} b_{1}+d_{1} b_{2}=d_{1} b_{1}+d_{2} b_{2}$ :

$$
\begin{aligned}
f^{\#} & =\frac{\left(a_{o}+d_{1} b_{1}\right) x_{1}^{2}+\left(2 a_{o}+d_{1} b_{1}+d_{2} b_{2}\right) x_{1} x_{2}+\left(a_{o}+d_{2} b_{2}\right) x_{2}^{2}}{b_{1} x_{1}+b_{2} x_{2}} \\
& =\frac{\left(a_{o}+d_{1} b_{1}\right) x_{1}^{2}+\left(a_{o}+d_{1} b_{1}\right) x_{1} x_{2}+\left(a_{o}+d_{2} b_{2}\right) x_{1} x_{2}+\left(a_{o}+d_{2} b_{2}\right) x_{2}^{2}}{b_{1} x_{1}+b_{2} x_{2}} \\
& =\frac{\left(a_{o}+d_{1} b_{1}\right)\left(x_{1}+x_{2}\right) x_{1}+\left(a_{o}+d_{2} b_{2}\right)\left(x_{1}+x_{2}\right) x_{2}}{b_{1} x_{1}+b_{2} x_{2}} \\
& =\frac{\left(a_{o}+d_{1} b_{1}\right) x_{1}+\left(a_{o}+d_{2} b_{2}\right) x_{2}}{b_{1} x_{1}+b_{2} x_{2}}
\end{aligned}
$$

This proves that the various Padè-based mixture models are interchangeable via linear composition shifts.

Figure S-2 provides an example of this interconnection for the different van Laar forms listed in Table 1 in the main text. Very different $b_{\text {i }}$ parameter values in the different forms can yield identical $g^{\mathrm{E}}$ functions. Table S-2 lists the equations implemented and the actual parameter values.

Table S-2. Parameter values for the van Laar expressions plotted in Figure S-2

\begin{tabular}{|l|r|l|r|l|r|}
\hline \multicolumn{2}{|c|}{$\mathrm{P}_{2}(2,1)$} & \multicolumn{2}{c|}{$\mathrm{P}_{2}(1,1)$} & \multicolumn{2}{c|}{$\mathrm{P}_{2}(0,1)$} \\
\hline$c_{11}$ & 1.000 & $c_{1}$ & -3.960 & $c_{\mathrm{o}}$ & -13.041 \\
\hline$c_{12}$ & 3.000 & $c_{2}$ & 0.002 & & \\
\hline$c_{22}$ & 2.000 & & & & \\
\hline$b_{1}$ & 1.000 & $b_{1}$ & 7.923 & $b_{1}$ & 19.565 \\
\hline$b_{2}$ & 4.000 & $b_{2}$ & 31.691 & $b_{2}$ & 78.259 \\
\hline$A_{21}$ & 1.500 & $A_{21}$ & 1.500 & $A_{21}$ & 1.500 \\
\hline$A_{12}$ & 0.375 & $A_{12}$ & 0.375 & $A_{12}$ & 0.375 \\
\hline
\end{tabular}



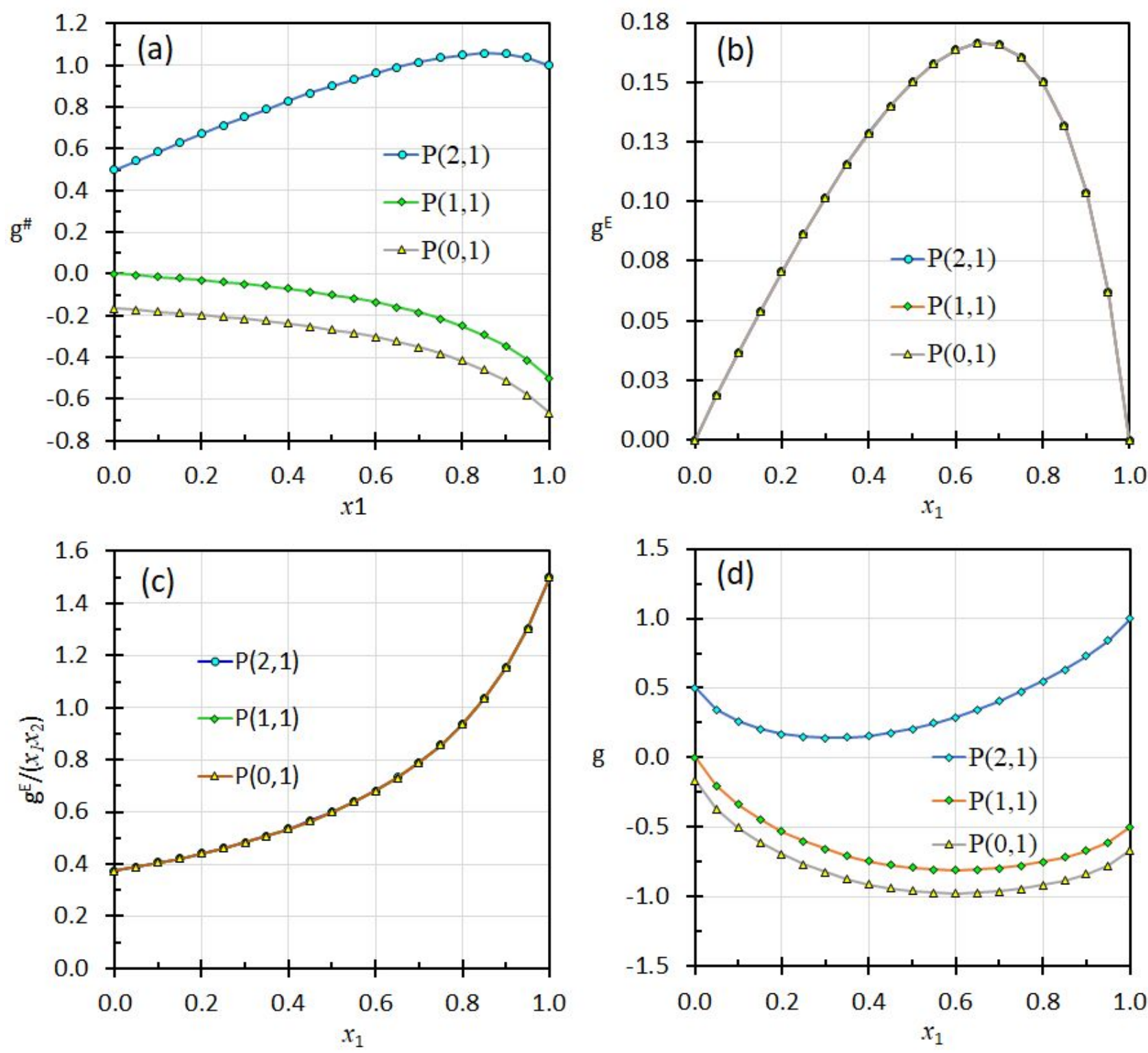

Figure S-4.1. Plots of $g^{\#}, g^{\mathrm{E}}, g^{\mathrm{E}} /\left(x_{1} x_{2}\right)$ and $g$ based on the parameter values presented in Table S-2 for different van Laar-type expressions. Despite considerable differences in $g^{\#}$ and $g$, the curves for $g^{\mathrm{E}}$ and $g^{\mathrm{E}} /\left(x_{1} x_{2}\right)$ coincide.

\section{References}

1. Bullen, P. S., Handbook of Means and their Inequalities. Kluwer Academic Publishers: Dortrecht: Netherlands, 2003.

2. Focke, W.; Sandrock, C.; Kok, S., Weighted-power-mean mixture model: Application to multicomponent liquid viscosity. Industrial \& Engineering Chemistry Research 2007, 46 (13), 4660-4666.

3. Scott, R. L., Corresponding States Treatment of Nonelectrolyte Solutions. Journal of Chemical Physics 1956, 25, 193. 
4. Focke, W. W., Mixture models based on neural network averaging. Neural Computation 2006, 18 (1), 1-9.

5. Focke, W. W.; Sandrock, C.; Kok, S., Weighted-power-mean mixture model: Application to multicomponent liquid viscosity. Industrial and Engineering Chemistry Research 2007, 46 (13), 4660-4666.

6. Michelsen , M. L.; Kistenmacher, H., On Composition-Dependent Interaction Coefficients. Fluid Phase Equilibria 1990, 58 (1-2), 229-230.

7. Oliver, E. D., Multicomponent Margules Equations. Industrial \& Engineering Chemistry Fundamentals 1968, 7 (2), 335-335.

8. Muggianu, Y.-M.; Gambino, M.; Bros, J.-P., Enthalpies de formation des alliages liquides bismuth-étain-gallium á $723 \mathrm{~K}$. Choix d'une représentation analytique des grandeurs d'excès intégrales et partielles. Journal de Chimie Physique 1975, 72, 83-88.

9. Hillert, M., Empirical methods of predicting and representing thermodynamic properties of ternary solution phases. Calphad 1980, 4 (1), 1-12.

10. Kattner, U. R., THE CALPHAD METHOD AND ITS ROLE IN MATERIAL AND PROCESS DEVELOPMENT. Tecnol Metal Mater Min 2016, 13 (1), 3-15.

11. Novák, J. P.; Matouš, J.; Pick, J., Liquid-liquid equilibria. Elsevier Science: Amsterdam, 1987; Vol. 7.

12. Behrens, D.; Eckermann, R., Vapor-Liquid Equilibrium Data Collection. DECHEMA: Frankfurt, 1977-1996. 


\section{S.4. Vapour-liquid data set}

Table S-2. Vapour-liquid equilibrium data set extracted from ${ }^{12}$

\begin{tabular}{|c|c|c|c|c|c|c|c|c|c|c|c|}
\hline \multirow[b]{2}{*}{$\#$} & \multicolumn{3}{|c|}{ Component } & \multicolumn{2}{|c|}{ Pressure } & \multicolumn{3}{|c|}{ Liquid composition } & \multicolumn{3}{|c|}{ Vapour composition } \\
\hline & 1 & 2 & 3 & $\mathrm{mmHg}$ & $\mathrm{kPa}$ & $x_{1}$ & $x_{2}$ & $x_{3}$ & $y_{1}$ & $y_{2}$ & $y_{3}$ \\
\hline 1 & Acetone & $\mathrm{CHCl} 3$ & Ethanol & 615.30 & 82.03 & 0.1000 & 0.8000 & 0.1000 & 0.0650 & 0.8150 & 0.1200 \\
\hline 2 & Acetone & $\mathrm{CHCl} 3$ & Ethanol & 612.70 & 81.69 & 0.1000 & 0.7000 & 0.2000 & 0.0630 & 0.7530 & 0.1840 \\
\hline 3 & Acetone & $\mathrm{CHCl} 3$ & Ethanol & 579.80 & 77.30 & 0.2000 & 0.7000 & 0.1000 & 0.1460 & 0.7400 & 0.1140 \\
\hline 4 & Acetone & $\mathrm{CHCl} 3$ & Ethanol & 600.80 & 80.10 & 0.1000 & 0.6000 & 0.3000 & 0.0710 & 0.7010 & 0.2280 \\
\hline 5 & Acetone & $\mathrm{CHCl} 3$ & Ethanol & 579.40 & 77.25 & 0.2000 & 0.6000 & 0.2000 & 0.1650 & 0.6580 & 0.1770 \\
\hline 6 & Acetone & $\mathrm{CHCl} 3$ & Ethanol & 565.60 & 75.41 & 0.3000 & 0.6000 & 0.1000 & 0.2650 & 0.6250 & 0.1100 \\
\hline 7 & Acetone & $\mathrm{CHCl} 3$ & Ethanol & 579.10 & 77.21 & 0.1000 & 0.5000 & 0.4000 & 0.0820 & 0.6510 & 0.2670 \\
\hline 8 & Acetone & $\mathrm{CHCl} 3$ & Ethanol & 565.40 & 75.38 & 0.2000 & 0.5000 & 0.3000 & 0.1820 & 0.5930 & 0.2250 \\
\hline 9 & Acetone & $\mathrm{CHCl} 3$ & Ethanol & 563.40 & 75.11 & 0.3000 & 0.5000 & 0.2000 & 0.2870 & 0.5360 & 0.1770 \\
\hline 10 & Acetone & $\mathrm{CHCl} 3$ & Ethanol & 562.10 & 74.94 & 0.4000 & 0.5000 & 0.1000 & 0.4200 & 0.4770 & 0.1030 \\
\hline 11 & Acetone & $\mathrm{CHCl} 3$ & Ethanol & 548.20 & 73.09 & 0.1000 & 0.4000 & 0.5000 & 0.0980 & 0.5930 & 0.3090 \\
\hline 12 & Acetone & $\mathrm{CHCl} 3$ & Ethanol & 540.10 & 72.01 & 0.2000 & 0.4000 & 0.4000 & 0.2070 & 0.5170 & 0.2760 \\
\hline 13 & Acetone & $\mathrm{CHCl} 3$ & Ethanol & 551.90 & 73.58 & 0.3000 & 0.4000 & 0.3000 & 0.3240 & 0.4490 & 0.2270 \\
\hline 14 & Acetone & $\mathrm{CHCl} 3$ & Ethanol & 562.50 & 74.99 & 0.4000 & 0.4000 & 0.2000 & 0.4400 & 0.3900 & 0.1700 \\
\hline 15 & Acetone & $\mathrm{CHCl} 3$ & Ethanol & 573.70 & 76.49 & 0.5000 & 0.4000 & 0.1000 & 0.5480 & 0.3520 & 0.1000 \\
\hline 16 & Acetone & $\mathrm{CHCl} 3$ & Ethanol & 523.60 & 69.81 & 0.1000 & 0.3000 & 0.6000 & 0.1220 & 0.5030 & 0.3750 \\
\hline 17 & Acetone & $\mathrm{CHCl} 3$ & Ethanol & 530.90 & 70.78 & 0.2000 & 0.3000 & 0.5000 & 0.2460 & 0.4250 & 0.3290 \\
\hline 18 & Acetone & $\mathrm{CHCl} 3$ & Ethanol & 533.80 & 71.17 & 0.3000 & 0.3000 & 0.4000 & 0.3740 & 0.3540 & 0.2720 \\
\hline 19 & Acetone & $\mathrm{CHCl} 3$ & Ethanol & 557.30 & 74.30 & 0.4000 & 0.3000 & 0.3000 & 0.4740 & 0.3070 & 0.2190 \\
\hline 20 & Acetone & $\mathrm{CHCl} 3$ & Ethanol & 577.50 & 76.99 & 0.5000 & 0.3000 & 0.2000 & 0.5810 & 0.2680 & 0.1510 \\
\hline 21 & Acetone & $\mathrm{CHCl} 3$ & Ethanol & 601.20 & 80.15 & 0.6000 & 0.3000 & 0.1000 & 0.6850 & 0.2290 & 0.0860 \\
\hline 22 & Acetone & $\mathrm{CHCl} 3$ & Ethanol & 478.00 & 63.73 & 0.1000 & 0.2000 & 0.7000 & 0.1630 & 0.3790 & 0.4580 \\
\hline
\end{tabular}




\begin{tabular}{|c|c|c|c|c|c|c|c|c|c|c|c|}
\hline 23 & Acetone & $\mathrm{CHCl} 3$ & Ethanol & 504.50 & 67.26 & 0.2000 & 0.2000 & 0.6000 & 0.2980 & 0.2980 & 0.4040 \\
\hline 24 & Acetone & $\mathrm{CHCl} 3$ & Ethanol & 524.50 & 69.93 & 0.3000 & 0.2000 & 0.5000 & 0.4310 & 0.2560 & 0.3130 \\
\hline 25 & Acetone & $\mathrm{CHCl} 3$ & Ethanol & 552.56 & 73.67 & 0.4000 & 0.2000 & 0.4000 & 0.5250 & 0.2110 & 0.2640 \\
\hline 26 & Acetone & $\mathrm{CHCl} 3$ & Ethanol & 577.60 & 77.01 & 0.5000 & 0.2000 & 0.3000 & 0.6200 & 0.1780 & 0.2020 \\
\hline 27 & Acetone & $\mathrm{CHCl} 3$ & Ethanol & 606.00 & 80.79 & 0.6000 & 0.2000 & 0.2000 & 0.7080 & 0.1570 & 0.1350 \\
\hline 28 & Acetone & $\mathrm{CHCl} 3$ & Ethanol & 623.60 & 83.14 & 0.7000 & 0.2000 & 0.1000 & 0.7980 & 0.1320 & 0.0700 \\
\hline 29 & Acetone & $\mathrm{CHCl} 3$ & Ethanol & 416.30 & 55.50 & 0.1000 & 0.1000 & 0.8000 & 0.2330 & 0.2040 & 0.5630 \\
\hline 30 & Acetone & $\mathrm{CHCl} 3$ & Ethanol & 463.70 & 61.82 & 0.2000 & 0.1000 & 0.7000 & 0.3640 & 0.1610 & 0.4750 \\
\hline 31 & Acetone & $\mathrm{CHCl} 3$ & Ethanol & 509.10 & 67.87 & 0.3000 & 0.1000 & 0.6000 & 0.5040 & 0.1290 & 0.3670 \\
\hline 32 & Acetone & $\mathrm{CHCl} 3$ & Ethanol & 550.00 & 73.33 & 0.4000 & 0.1000 & 0.5000 & 0.5880 & 0.1060 & 0.3060 \\
\hline 33 & Acetone & $\mathrm{CHCl} 3$ & Ethanol & 583.80 & 77.83 & 0.5000 & 0.1000 & 0.4000 & 0.6620 & 0.0940 & 0.2440 \\
\hline 34 & Acetone & $\mathrm{CHCl} 3$ & Ethanol & 618.50 & 82.46 & 0.6000 & 0.1000 & 0.3000 & 0.7350 & 0.0850 & 0.1800 \\
\hline 35 & Acetone & $\mathrm{CHCl} 3$ & Ethanol & 643.20 & 85.75 & 0.7000 & 0.1000 & 0.2000 & 0.8070 & 0.0730 & 0.1200 \\
\hline 36 & Acetone & $\mathrm{CHCl} 3$ & Ethanol & 665.00 & 88.66 & 0.8000 & 0.1000 & 0.1000 & 0.8780 & 0.0560 & 0.0660 \\
\hline 37 & Acetone & $\mathrm{CHCl} 3$ & & 590.80 & 78.77 & 0.1000 & 0.9000 & 0.0000 & 0.0670 & 0.9330 & 0.0000 \\
\hline 38 & Acetone & $\mathrm{CHCl} 3$ & & 565.50 & 75.39 & 0.1990 & 0.8010 & 0.0000 & 0.1560 & 0.8440 & 0.0000 \\
\hline 39 & Acetone & $\mathrm{CHCl} 3$ & & 553.10 & 73.74 & 0.3000 & 0.7000 & 0.0000 & 0.2720 & 0.7280 & 0.0000 \\
\hline 40 & Acetone & $\mathrm{CHCl} 3$ & & 546.00 & 72.79 & 0.4050 & 0.5950 & 0.0000 & 0.4190 & 0.5810 & 0.0000 \\
\hline 41 & Acetone & $\mathrm{CHCl} 3$ & & 557.80 & 74.37 & 0.5000 & 0.5000 & 0.0000 & 0.5550 & 0.4450 & 0.0000 \\
\hline 42 & Acetone & $\mathrm{CHCl} 3$ & & 577.70 & 77.02 & 0.5890 & 0.4110 & 0.0000 & 0.6680 & 0.3320 & 0.0000 \\
\hline 43 & Acetone & $\mathrm{CHCl} 3$ & & 610.60 & 81.41 & 0.6890 & 0.3110 & 0.0000 & 0.7790 & 0.2210 & 0.0000 \\
\hline 44 & Acetone & $\mathrm{CHCl} 3$ & & 655.50 & 87.39 & 0.7980 & 0.2020 & 0.0000 & 0.8870 & 0.1130 & 0.0000 \\
\hline 45 & Acetone & $\mathrm{CHCl} 3$ & & 689.90 & 91.98 & 0.8950 & 0.1050 & 0.0000 & 0.9440 & 0.0560 & 0.0000 \\
\hline 46 & Acetone & & Ethanol & 375.16 & 50.02 & 0.1000 & 0.0000 & 0.9000 & 0.3194 & 0.0000 & 0.6806 \\
\hline 47 & Acetone & & Ethanol & 447.09 & 59.61 & 0.2000 & 0.0000 & 0.8000 & 0.4815 & 0.0000 & 0.5185 \\
\hline 48 & Acetone & & Ethanol & 504.27 & 67.23 & 0.3000 & 0.0000 & 0.7000 & 0.5849 & 0.0000 & 0.4151 \\
\hline 49 & Acetone & & Ethanol & 551.45 & 73.52 & 0.4000 & 0.0000 & 0.6000 & 0.6606 & 0.0000 & 0.3394 \\
\hline
\end{tabular}




\begin{tabular}{|c|c|c|c|c|c|c|c|c|c|c|c|}
\hline 50 & Acetone & & Ethanol & 591.54 & 78.87 & 0.5000 & 0.0000 & 0.5000 & 0.7218 & 0.0000 & 0.2782 \\
\hline 51 & Acetone & & Ethanol & 626.43 & 83.52 & 0.6000 & 0.0000 & 0.4000 & 0.7753 & 0.0000 & 0.2247 \\
\hline 52 & Acetone & & Ethanol & 657.41 & 87.65 & 0.7000 & 0.0000 & 0.3000 & 0.8257 & 0.0000 & 0.1743 \\
\hline 53 & Acetone & & Ethanol & 685.30 & 91.37 & 0.8000 & 0.0000 & 0.2000 & 0.8768 & 0.0000 & 0.1232 \\
\hline 54 & Acetone & & Ethanol & 710.33 & 94.70 & 0.9000 & 0.0000 & 0.1000 & 0.9330 & 0.0000 & 0.0670 \\
\hline 55 & & $\mathrm{CHCl} 3$ & Ethanol & 306.38 & 40.85 & 0.0000 & 0.0331 & 0.9669 & 0.0000 & 0.1162 & 0.8838 \\
\hline 56 & & $\mathrm{CHCl} 3$ & Ethanol & 339.89 & 45.31 & 0.0000 & 0.0712 & 0.9288 & 0.0000 & 0.2302 & 0.7698 \\
\hline 57 & & $\mathrm{CHCl} 3$ & Ethanol & 346.89 & 46.25 & 0.0000 & 0.0802 & 0.9198 & 0.0000 & 0.2533 & 0.7467 \\
\hline 58 & & $\mathrm{CHCl} 3$ & Ethanol & 367.01 & 48.93 & 0.0000 & 0.1029 & 0.8971 & 0.0000 & 0.3123 & 0.6877 \\
\hline 59 & & $\mathrm{CHCl} 3$ & Ethanol & 407.90 & 54.38 & 0.0000 & 0.1479 & 0.8521 & 0.0000 & 0.4035 & 0.5965 \\
\hline 60 & & $\mathrm{CHCl} 3$ & Ethanol & 441.04 & 58.80 & 0.0000 & 0.1869 & 0.8131 & 0.0000 & 0.4795 & 0.5205 \\
\hline 61 & & $\mathrm{CHCl} 3$ & Ethanol & 469.41 & 62.58 & 0.0000 & 0.2201 & 0.7799 & 0.0000 & 0.5271 & 0.4729 \\
\hline 62 & & $\mathrm{CHCl} 3$ & Ethanol & 508.78 & 67.83 & 0.0000 & 0.2806 & 0.7194 & 0.0000 & 0.5942 & 0.4058 \\
\hline 63 & & $\mathrm{CHCl} 3$ & Ethanol & 543.53 & 72.46 & 0.0000 & 0.3412 & 0.6588 & 0.0000 & 0.6407 & 0.3593 \\
\hline 64 & & $\mathrm{CHCl} 3$ & Ethanol & 545.72 & 72.76 & 0.0000 & 0.3445 & 0.6555 & 0.0000 & 0.6419 & 0.3581 \\
\hline 65 & & $\mathrm{CHCl} 3$ & Ethanol & 560.25 & 74.69 & 0.0000 & 0.3767 & 0.6233 & 0.0000 & 0.6641 & 0.3359 \\
\hline 66 & & $\mathrm{CHCl} 3$ & Ethanol & 566.74 & 75.56 & 0.0000 & 0.3904 & 0.6096 & 0.0000 & 0.6720 & 0.3280 \\
\hline 67 & & $\mathrm{CHCl} 3$ & Ethanol & 569.02 & 75.86 & 0.0000 & 0.3965 & 0.6035 & 0.0000 & 0.6760 & 0.3240 \\
\hline 68 & & $\mathrm{CHCl} 3$ & Ethanol & 599.03 & 79.86 & 0.0000 & 0.4794 & 0.5206 & 0.0000 & 0.7161 & 0.2839 \\
\hline 69 & & $\mathrm{CHCl} 3$ & Ethanol & 623.67 & 83.15 & 0.0000 & 0.5730 & 0.4270 & 0.0000 & 0.7527 & 0.2473 \\
\hline 70 & & $\mathrm{CHCl} 3$ & Ethanol & 632.14 & 84.28 & 0.0000 & 0.6211 & 0.3789 & 0.0000 & 0.7639 & 0.2361 \\
\hline 71 & & $\mathrm{CHCl} 3$ & Ethanol & 641.49 & 85.52 & 0.0000 & 0.6851 & 0.3149 & 0.0000 & 0.7857 & 0.2143 \\
\hline 72 & & $\mathrm{CHCl} 3$ & Ethanol & 646.79 & 86.23 & 0.0000 & 0.7269 & 0.2731 & 0.0000 & 0.8010 & 0.1990 \\
\hline 73 & & $\mathrm{CHCl} 3$ & Ethanol & 650.96 & 86.79 & 0.0000 & 0.7764 & 0.2236 & 0.0000 & 0.8181 & 0.1819 \\
\hline 74 & & $\mathrm{CHCl} 3$ & Ethanol & 653.11 & 87.07 & 0.0000 & 0.8390 & 0.1610 & 0.0000 & 0.8417 & 0.1583 \\
\hline 75 & & $\mathrm{CHCl} 3$ & Ethanol & 650.38 & 86.71 & 0.0000 & 0.9037 & 0.0963 & 0.0000 & 0.8798 & 0.1202 \\
\hline 76 & & $\mathrm{CHCl} 3$ & Ethanol & 644.24 & 85.89 & 0.0000 & 0.9430 & 0.0570 & 0.0000 & 0.9150 & 0.0850 \\
\hline
\end{tabular}




\begin{tabular}{|c|c|c|c|c|c|c|c|c|c|c|c|}
\hline 77 & & $\mathrm{CHCl} 3$ & Ethanol & 626.79 & 83.57 & 0.0000 & 0.9652 & 0.0348 & 0.0000 & 0.9408 & 0.0592 \\
\hline 78 & Acetone & Methanol & Cyclohexane & 822.00 & 109.59 & 0.1130 & 0.1260 & 0.7610 & 0.2610 & 0.3840 & 0.3550 \\
\hline 79 & Acetone & Methanol & Cyclohexane & 838.00 & 111.72 & 0.1160 & 0.2450 & 0.6390 & 0.2030 & 0.4470 & 0.3500 \\
\hline 80 & Acetone & Methanol & Cyclohexane & 839.00 & 111.86 & 0.1160 & 0.3790 & 0.5050 & 0.1770 & 0.4730 & 0.3500 \\
\hline 81 & Acetone & Methanol & Cyclohexane & 838.00 & 111.72 & 0.1210 & 0.5010 & 0.3780 & 0.1620 & 0.4870 & 0.3510 \\
\hline 82 & Acetone & Methanol & Cyclohexane & 835.00 & 111.32 & 0.1240 & 0.6340 & 0.2420 & 0.1590 & 0.4990 & 0.3420 \\
\hline 83 & Acetone & Methanol & Cyclohexane & 810.00 & 107.99 & 0.1240 & 0.7560 & 0.1200 & 0.1650 & 0.5320 & 0.3030 \\
\hline 84 & Acetone & Methanol & Cyclohexane & 839.00 & 111.86 & 0.2430 & 0.1150 & 0.6420 & 0.4020 & 0.2710 & 0.3270 \\
\hline 85 & Acetone & Methanol & Cyclohexane & 858.00 & 114.39 & 0.2440 & 0.2520 & 0.5040 & 0.3200 & 0.3610 & 0.3190 \\
\hline 86 & Acetone & Methanol & Cyclohexane & 861.00 & 114.79 & 0.2520 & 0.3750 & 0.3730 & 0.2920 & 0.3930 & 0.3150 \\
\hline 87 & Acetone & Methanol & Cyclohexane & 859.00 & 114.52 & 0.2560 & 0.4950 & 0.2490 & 0.2790 & 0.4130 & 0.3080 \\
\hline 88 & Acetone & Methanol & Cyclohexane & 835.00 & 111.32 & 0.2490 & 0.6300 & 0.1210 & 0.2960 & 0.4450 & 0.2590 \\
\hline 89 & Acetone & Methanol & Cyclohexane & 847.00 & 112.92 & 0.3760 & 0.1220 & 0.5020 & 0.4750 & 0.2140 & 0.3110 \\
\hline 90 & Acetone & Methanol & Cyclohexane & 864.00 & 115.19 & 0.3840 & 0.2350 & 0.3810 & 0.4270 & 0.2790 & 0.2940 \\
\hline 91 & Acetone & Methanol & Cyclohexane & 868.00 & 115.72 & 0.4070 & 0.3730 & 0.2200 & 0.3960 & 0.3240 & 0.2800 \\
\hline 92 & Acetone & Methanol & Cyclohexane & 848.00 & 113.06 & 0.3800 & 0.5000 & 0.1200 & 0.3920 & 0.3820 & 0.2260 \\
\hline 93 & Acetone & Methanol & Cyclohexane & 851.00 & 113.46 & 0.5280 & 0.0900 & 0.3820 & 0.5800 & 0.1370 & 0.2830 \\
\hline 94 & Acetone & Methanol & Cyclohexane & 865.00 & 115.32 & 0.5370 & 0.2140 & 0.2490 & 0.5090 & 0.2270 & 0.2640 \\
\hline 95 & Acetone & Methanol & Cyclohexane & 852.00 & 113.59 & 0.5150 & 0.3650 & 0.1200 & 0.5080 & 0.2780 & 0.2140 \\
\hline 96 & Acetone & Methanol & Cyclohexane & 858.00 & 114.39 & 0.6750 & 0.0740 & 0.2510 & 0.6550 & 0.0950 & 0.2500 \\
\hline 97 & Acetone & Methanol & Cyclohexane & 845.00 & 112.66 & 0.6610 & 0.2180 & 0.1210 & 0.6170 & 0.1870 & 0.1960 \\
\hline 98 & Acetone & Methanol & Cyclohexane & 829.00 & 110.52 & 0.8260 & 0.0520 & 0.1220 & 0.7480 & 0.0720 & 0.1800 \\
\hline 99 & Acetone & Methanol & & 542.17 & 72.28 & 0.0287 & 0.9713 & 0.0000 & 0.0647 & 0.9353 & 0.0000 \\
\hline 100 & Acetone & Methanol & & 564.61 & 75.28 & 0.0570 & 0.9430 & 0.0000 & 0.1295 & 0.8705 & 0.0000 \\
\hline 101 & Acetone & Methanol & & 569.56 & 75.94 & 0.0644 & 0.9356 & 0.0000 & 0.1407 & 0.8593 & 0.0000 \\
\hline 102 & Acetone & Methanol & & 581.45 & 77.52 & 0.0858 & 0.9142 & 0.0000 & 0.1848 & 0.8152 & 0.0000 \\
\hline 103 & Acetone & Methanol & & 592.15 & 78.95 & 0.1046 & 0.8954 & 0.0000 & 0.2190 & 0.7810 & 0.0000 \\
\hline
\end{tabular}




\begin{tabular}{|c|c|c|c|c|c|c|c|c|c|c|c|}
\hline 104 & Acetone & Methanol & & 610.13 & 81.34 & 0.1357 & 0.8643 & 0.0000 & 0.2637 & 0.7363 & 0.0000 \\
\hline 105 & Acetone & Methanol & & 618.98 & 82.52 & 0.1452 & 0.8548 & 0.0000 & 0.2694 & 0.7306 & 0.0000 \\
\hline 106 & Acetone & Methanol & & 628.16 & 83.75 & 0.1663 & 0.8337 & 0.0000 & 0.3055 & 0.6945 & 0.0000 \\
\hline 107 & Acetone & Methanol & & 650.74 & 86.76 & 0.2173 & 0.7827 & 0.0000 & 0.3633 & 0.6367 & 0.0000 \\
\hline 108 & Acetone & Methanol & & 657.70 & 87.69 & 0.2390 & 0.7610 & 0.0000 & 0.3863 & 0.6137 & 0.0000 \\
\hline 109 & Acetone & Methanol & & 675.68 & 90.08 & 0.2787 & 0.7213 & 0.0000 & 0.4184 & 0.5816 & 0.0000 \\
\hline 110 & Acetone & Methanol & & 699.07 & 93.20 & 0.3579 & 0.6421 & 0.0000 & 0.4779 & 0.5221 & 0.0000 \\
\hline 111 & Acetone & Methanol & & 712.65 & 95.01 & 0.4050 & 0.5950 & 0.0000 & 0.5135 & 0.4865 & 0.0000 \\
\hline 112 & Acetone & Methanol & & 722.76 & 96.36 & 0.4480 & 0.5520 & 0.0000 & 0.5512 & 0.4488 & 0.0000 \\
\hline 113 & Acetone & Methanol & & 732.37 & 97.64 & 0.5052 & 0.4948 & 0.0000 & 0.5844 & 0.4156 & 0.0000 \\
\hline 114 & Acetone & Methanol & & 738.49 & 98.46 & 0.5432 & 0.4568 & 0.0000 & 0.6174 & 0.3826 & 0.0000 \\
\hline 115 & Acetone & Methanol & & 748.61 & 99.81 & 0.6332 & 0.3668 & 0.0000 & 0.6772 & 0.3228 & 0.0000 \\
\hline 116 & Acetone & Methanol & & 752.18 & 100.28 & 0.6538 & 0.3462 & 0.0000 & 0.6849 & 0.3151 & 0.0000 \\
\hline 117 & Acetone & Methanol & & 749.65 & 99.95 & 0.6605 & 0.3395 & 0.0000 & 0.6926 & 0.3074 & 0.0000 \\
\hline 118 & Acetone & Methanol & & 752.11 & 100.27 & 0.6945 & 0.3055 & 0.0000 & 0.7124 & 0.2876 & 0.0000 \\
\hline 119 & Acetone & Methanol & & 753.53 & 100.46 & 0.7327 & 0.2673 & 0.0000 & 0.7383 & 0.2617 & 0.0000 \\
\hline 120 & Acetone & Methanol & & 753.85 & 100.51 & 0.7525 & 0.2475 & 0.0000 & 0.7618 & 0.2382 & 0.0000 \\
\hline 121 & Acetone & Methanol & & 757.52 & 100.99 & 0.7752 & 0.2248 & 0.0000 & 0.7729 & 0.2271 & 0.0000 \\
\hline 122 & Acetone & Methanol & & 757.97 & 101.05 & 0.7922 & 0.2078 & 0.0000 & 0.7876 & 0.2124 & 0.0000 \\
\hline 123 & Acetone & Methanol & & 749.10 & 99.87 & 0.9080 & 0.0920 & 0.0000 & 0.8959 & 0.1041 & 0.0000 \\
\hline 124 & Acetone & Methanol & & 750.31 & 100.03 & 0.9088 & 0.0912 & 0.0000 & 0.8963 & 0.1037 & 0.0000 \\
\hline 125 & Acetone & Methanol & & 750.47 & 100.05 & 0.9197 & 0.0803 & 0.0000 & 0.8941 & 0.1059 & 0.0000 \\
\hline 126 & Acetone & Methanol & & 748.52 & 99.79 & 0.9448 & 0.0552 & 0.0000 & 0.9336 & 0.0664 & 0.0000 \\
\hline 127 & Acetone & & Cyclohexane & 560.60 & 74.74 & 0.0900 & 0.0000 & 0.9100 & 0.4320 & 0.0000 & 0.5680 \\
\hline 128 & Acetone & & Cyclohexane & 683.30 & 91.10 & 0.1950 & 0.0000 & 0.8050 & 0.5760 & 0.0000 & 0.4240 \\
\hline 129 & Acetone & & Cyclohexane & 729.10 & 97.21 & 0.2780 & 0.0000 & 0.7220 & 0.6230 & 0.0000 & 0.3770 \\
\hline 130 & Acetone & & Cyclohexane & 752.70 & 100.35 & 0.3800 & 0.0000 & 0.6200 & 0.6500 & 0.0000 & 0.3500 \\
\hline
\end{tabular}




\begin{tabular}{|c|c|c|c|c|c|c|c|c|c|c|c|}
\hline 131 & Acetone & & Cyclohexane & 777.30 & 103.63 & 0.4930 & 0.0000 & 0.5070 & 0.6770 & 0.0000 & 0.3230 \\
\hline 132 & Acetone & & Cyclohexane & 789.00 & 105.19 & 0.5980 & 0.0000 & 0.4020 & 0.7030 & 0.0000 & 0.2970 \\
\hline 133 & Acetone & & Cyclohexane & 800.10 & 106.67 & 0.7000 & 0.0000 & 0.3000 & 0.7380 & 0.0000 & 0.2620 \\
\hline 134 & Acetone & & Cyclohexane & 802.30 & 106.96 & 0.7700 & 0.0000 & 0.2300 & 0.7660 & 0.0000 & 0.2340 \\
\hline 135 & Acetone & & Cyclohexane & 784.60 & 104.60 & 0.8950 & 0.0000 & 0.1050 & 0.8650 & 0.0000 & 0.1350 \\
\hline 136 & & Methanol & Cyclohexane & 544.00 & 72.53 & 0.0000 & 0.0266 & 0.9734 & 0.0000 & 0.4248 & 0.5752 \\
\hline 137 & & Methanol & Cyclohexane & 679.00 & 90.53 & 0.0000 & 0.0497 & 0.9503 & 0.0000 & 0.5290 & 0.4710 \\
\hline 138 & & Methanol & Cyclohexane & 733.00 & 97.73 & 0.0000 & 0.0715 & 0.9285 & 0.0000 & 0.5740 & 0.4260 \\
\hline 139 & & Methanol & Cyclohexane & 756.00 & 100.79 & 0.0000 & 0.0980 & 0.9020 & 0.0000 & 0.6005 & 0.3995 \\
\hline 140 & & Methanol & Cyclohexane & 757.00 & 100.93 & 0.0000 & 0.8922 & 0.1078 & 0.0000 & 0.6398 & 0.3602 \\
\hline 141 & & Methanol & Cyclohexane & 741.00 & 98.79 & 0.0000 & 0.9144 & 0.0856 & 0.0000 & 0.6595 & 0.3405 \\
\hline 142 & & Methanol & Cyclohexane & 724.00 & 96.53 & 0.0000 & 0.9291 & 0.0709 & 0.0000 & 0.6789 & 0.3211 \\
\hline 143 & & Methanol & Cyclohexane & 701.00 & 93.46 & 0.0000 & 0.9425 & 0.0575 & 0.0000 & 0.7032 & 0.2968 \\
\hline 144 & & Methanol & Cyclohexane & 661.00 & 88.13 & 0.0000 & 0.9601 & 0.0399 & 0.0000 & 0.7492 & 0.2508 \\
\hline 145 & & Methanol & Cyclohexane & 624.00 & 83.19 & 0.0000 & 0.9731 & 0.0269 & 0.0000 & 0.7999 & 0.2001 \\
\hline 146 & & Methanol & Cyclohexane & 572.00 & 76.26 & 0.0000 & 0.9883 & 0.0117 & 0.0000 & 0.8905 & 0.1095 \\
\hline 147 & Acetonitrile & Benzene & $\mathrm{CCl} 4$ & 286.00 & 38.13 & 0.1510 & 0.5980 & 0.2510 & 0.2250 & 0.5010 & 0.2740 \\
\hline 148 & Acetonitrile & Benzene & $\mathrm{CCl} 4$ & 272.00 & 36.26 & 0.1560 & 0.7670 & 0.0770 & 0.2560 & 0.6500 & 0.0940 \\
\hline 149 & Acetonitrile & Benzene & $\mathrm{CCl} 4$ & 336.30 & 44.84 & 0.1690 & 0.1790 & 0.6520 & 0.3030 & 0.1260 & 0.5710 \\
\hline 150 & Acetonitrile & Benzene & $\mathrm{CCl} 4$ & 300.10 & 40.01 & 0.1760 & 0.5110 & 0.3130 & 0.2690 & 0.3960 & 0.3350 \\
\hline 151 & Acetonitrile & Benzene & $\mathrm{CCl} 4$ & 282.50 & 37.66 & 0.2790 & 0.6570 & 0.0640 & 0.3520 & 0.5640 & 0.0840 \\
\hline 152 & Acetonitrile & Benzene & $\mathrm{CCl} 4$ & 300.90 & 40.12 & 0.2890 & 0.5060 & 0.2050 & 0.3540 & 0.4030 & 0.2430 \\
\hline 153 & Acetonitrile & Benzene & $\mathrm{CCl} 4$ & 312.00 & 41.60 & 0.3030 & 0.4360 & 0.2610 & 0.3600 & 0.3380 & 0.3020 \\
\hline 154 & Acetonitrile & Benzene & $\mathrm{CCl} 4$ & 347.80 & 46.37 & 0.3040 & 0.1540 & 0.5420 & 0.3740 & 0.1070 & 0.5190 \\
\hline 155 & Acetonitrile & Benzene & $\mathrm{CCl} 4$ & 337.20 & 44.96 & 0.3100 & 0.2480 & 0.4420 & 0.3650 & 0.1800 & 0.4550 \\
\hline 156 & Acetonitrile & Benzene & $\mathrm{CCl} 4$ & 323.40 & 43.12 & 0.3720 & 0.3560 & 0.2720 & 0.3910 & 0.2800 & 0.3290 \\
\hline 157 & Acetonitrile & Benzene & $\mathrm{CCl} 4$ & 287.10 & 38.28 & 0.3950 & 0.5550 & 0.0500 & 0.4220 & 0.4990 & 0.0790 \\
\hline
\end{tabular}




\begin{tabular}{|c|c|c|c|c|c|c|c|c|c|c|c|}
\hline 158 & Acetonitrile & Benzene & $\mathrm{CCl} 4$ & 303.80 & 40.50 & 0.3960 & 0.4340 & 0.1700 & 0.4020 & 0.3640 & 0.2340 \\
\hline 159 & Acetonitrile & Benzene & $\mathrm{CCl} 4$ & 363.20 & 48.42 & 0.4010 & 0.0700 & 0.5290 & 0.4020 & 0.0500 & 0.5480 \\
\hline 160 & Acetonitrile & Benzene & $\mathrm{CCl} 4$ & 315.30 & 42.04 & 0.4040 & 0.3790 & 0.2170 & 0.4080 & 0.3080 & 0.2840 \\
\hline 161 & Acetonitrile & Benzene & $\mathrm{CCl} 4$ & 350.10 & 46.68 & 0.4220 & 0.1280 & 0.4500 & 0.3930 & 0.1030 & 0.5040 \\
\hline 162 & Acetonitrile & Benzene & $\mathrm{CCl} 4$ & 339.70 & 45.29 & 0.4230 & 0.2080 & 0.3690 & 0.4120 & 0.1590 & 0.4290 \\
\hline 163 & Acetonitrile & Benzene & $\mathrm{CCl} 4$ & 303.90 & 40.52 & 0.4790 & 0.3790 & 0.1420 & 0.4390 & 0.3450 & 0.2160 \\
\hline 164 & Acetonitrile & Benzene & $\mathrm{CCl} 4$ & 350.10 & 46.68 & 0.4910 & 0.1140 & 0.3950 & 0.4220 & 0.0870 & 0.4910 \\
\hline 165 & Acetonitrile & Benzene & $\mathrm{CCl} 4$ & 315.00 & 42.00 & 0.4940 & 0.3250 & 0.1810 & 0.4410 & 0.2860 & 0.2730 \\
\hline 166 & Acetonitrile & Benzene & $\mathrm{CCl} 4$ & 364.60 & 48.61 & 0.4970 & 0.0560 & 0.4470 & 0.4270 & 0.0430 & 0.5300 \\
\hline 167 & Acetonitrile & Benzene & $\mathrm{CCl} 4$ & 287.80 & 38.37 & 0.5050 & 0.4530 & 0.0420 & 0.4780 & 0.4520 & 0.0700 \\
\hline 68 & Acetonitrile & Benzene & $\mathrm{CCl} 4$ & 339.60 & 45.28 & 0.5100 & 0.1830 & 0.3070 & 0.4440 & 0.1500 & 0.4060 \\
\hline 69 & Acetonitrile & Benzene & $\mathrm{CCl} 4$ & 323.80 & 43.17 & 0.5180 & 0.2790 & 0.2030 & 0.4520 & 0.2420 & 0.3060 \\
\hline 70 & Acetonitrile & Benzene & $\mathrm{CCl} 4$ & 302.30 & 40.30 & 0.5530 & 0.3280 & 0.1190 & 0.4720 & 0.3200 & 0.2080 \\
\hline 171 & Acetonitrile & Benzene & $\mathrm{CCl} 4$ & 347.90 & 46.38 & 0.5630 & 0.0990 & 0.3380 & 0.4430 & 0.0840 & 0.4730 \\
\hline 172 & Acetonitrile & Benzene & $\mathrm{CCl} 4$ & 286.00 & 38.13 & 0.5700 & 0.3960 & 0.0340 & 0.5150 & 0.4210 & 0.0640 \\
\hline 173 & Acetonitrile & Benzene & $\mathrm{CCl} 4$ & 311.60 & 41.54 & 0.5750 & 0.2760 & 0.1490 & 0.4810 & 0.2650 & 0.2540 \\
\hline 174 & Acetonitrile & Benzene & $\mathrm{CCl} 4$ & 337.00 & 44.93 & 0.5780 & 0.1590 & 0.2630 & 0.4680 & 0.1400 & 0.3920 \\
\hline 175 & Acetonitrile & Benzene & $\mathrm{CCl} 4$ & 363.20 & 48.42 & 0.5930 & 0.0480 & 0.3590 & 0.4570 & 0.0390 & 0.5040 \\
\hline 176 & Acetonitrile & Benzene & $\mathrm{CCl} 4$ & 318.90 & 42.52 & 0.6040 & 0.2330 & 0.1630 & 0.4550 & 0.2280 & 0.3170 \\
\hline 177 & Acetonitrile & Benzene & $\mathrm{CCl} 4$ & 298.20 & 39.76 & 0.6290 & 0.2760 & 0.0950 & 0.5120 & 0.2980 & 0.1900 \\
\hline 178 & Acetonitrile & Benzene & $\mathrm{CCl} 4$ & 342.30 & 45.64 & 0.6400 & 0.0830 & 0.2770 & 0.4710 & 0.0800 & 0.4490 \\
\hline 179 & Acetonitrile & Benzene & $\mathrm{CCl} 4$ & 281.60 & 37.54 & 0.6400 & 0.3330 & 0.0270 & 0.5570 & 0.3850 & 0.0580 \\
\hline 180 & Acetonitrile & Benzene & $\mathrm{CCl} 4$ & 307.40 & 40.98 & 0.6420 & 0.2370 & 0.1210 & 0.5140 & 0.2490 & 0.2370 \\
\hline 181 & Acetonitrile & Benzene & $\mathrm{CCl} 4$ & 331.90 & 44.25 & 0.6460 & 0.1330 & 0.2210 & 0.4900 & 0.1320 & 0.3780 \\
\hline 182 & Acetonitrile & Benzene & $\mathrm{CCl} 4$ & 314.40 & 41.92 & 0.6590 & 0.2040 & 0.1370 & 0.5120 & 0.2160 & 0.2720 \\
\hline 183 & Acetonitrile & Benzene & $\mathrm{CCl} 4$ & 357.10 & 47.61 & 0.6620 & 0.0410 & 0.2970 & 0.4770 & 0.0380 & 0.4850 \\
\hline 184 & Acetonitrile & Benzene & $\mathrm{CCl} 4$ & 294.00 & 39.20 & 0.6730 & 0.2440 & 0.0830 & 0.5470 & 0.2780 & 0.1750 \\
\hline
\end{tabular}




\begin{tabular}{|c|c|c|c|c|c|c|c|c|c|c|c|}
\hline 185 & Acetonitrile & Benzene & $\mathrm{CCl} 4$ & 277.30 & 36.97 & 0.7050 & 0.2750 & 0.0200 & 0.6000 & 0.3490 & 0.0510 \\
\hline 186 & Acetonitrile & Benzene & $\mathrm{CCl} 4$ & 300.50 & 40.06 & 0.7060 & 0.1980 & 0.0960 & 0.5480 & 0.2350 & 0.2170 \\
\hline 187 & Acetonitrile & Benzene & $\mathrm{CCl} 4$ & 324.40 & 43.25 & 0.7090 & 0.1130 & 0.1780 & 0.5260 & 0.1240 & 0.3500 \\
\hline 188 & Acetonitrile & Benzene & $\mathrm{CCl} 4$ & 306.80 & 40.90 & 0.7120 & 0.1750 & 0.1130 & 0.5410 & 0.2030 & 0.2560 \\
\hline 189 & Acetonitrile & Benzene & $\mathrm{CCl} 4$ & 289.00 & 38.53 & 0.7210 & 0.2100 & 0.0690 & 0.5690 & 0.2680 & 0.1630 \\
\hline 190 & Acetonitrile & Benzene & $\mathrm{CCl} 4$ & 347.80 & 46.37 & 0.7310 & 0.0360 & 0.2330 & 0.5050 & 0.0350 & 0.4600 \\
\hline 191 & Acetonitrile & Benzene & $\mathrm{CCl} 4$ & 328.20 & 43.76 & 0.7500 & 0.0640 & 0.1860 & 0.5250 & 0.0730 & 0.4020 \\
\hline 192 & Acetonitrile & Benzene & $\mathrm{CCl} 4$ & 293.40 & 39.12 & 0.7530 & 0.1690 & 0.0780 & 0.5840 & 0.2200 & 0.1960 \\
\hline 193 & Acetonitrile & Benzene & $\mathrm{CCl} 4$ & 317.40 & 42.32 & 0.7560 & 0.0970 & 0.1470 & 0.5450 & 0.1210 & 0.3340 \\
\hline 194 & Acetonitrile & Benzene & $\mathrm{CCl} 4$ & 271.40 & 36.18 & 0.7570 & 0.2270 & 0.0160 & 0.6330 & 0.3220 & 0.0450 \\
\hline 195 & Acetonitrile & Benzene & $\mathrm{CCl} 4$ & 282.50 & 37.66 & 0.7630 & 0.1810 & 0.0560 & 0.5960 & 0.2520 & 0.1520 \\
\hline 196 & Acetonitrile & Benzene & $\mathrm{CCl} 4$ & 299.40 & 39.92 & 0.7700 & 0.1430 & 0.0870 & 0.5890 & 0.1880 & 0.2230 \\
\hline 197 & Acetonitrile & Benzene & $\mathrm{CCl} 4$ & 339.20 & 45.22 & 0.7820 & 0.0270 & 0.1910 & 0.5330 & 0.0340 & 0.4330 \\
\hline 198 & Acetonitrile & Benzene & $\mathrm{CCl} 4$ & 319.50 & 42.60 & 0.7910 & 0.0570 & 0.1520 & 0.5480 & 0.0690 & 0.3830 \\
\hline 199 & Acetonitrile & Benzene & $\mathrm{CCl} 4$ & 286.40 & 38.18 & 0.7910 & 0.1470 & 0.0620 & 0.6100 & 0.2090 & 0.1810 \\
\hline 200 & Acetonitrile & Benzene & $\mathrm{CCl} 4$ & 307.10 & 40.94 & 0.7990 & 0.0840 & 0.1170 & 0.5860 & 0.1150 & 0.2990 \\
\hline 201 & Acetonitrile & Benzene & $\mathrm{CCl} 4$ & 265.50 & 35.40 & 0.8000 & 0.1880 & 0.0120 & 0.6650 & 0.2960 & 0.0390 \\
\hline 202 & Acetonitrile & Benzene & $\mathrm{CCl} 4$ & 288.20 & 38.42 & 0.8140 & 0.1200 & 0.0660 & 0.6250 & 0.1730 & 0.2020 \\
\hline 203 & Acetonitrile & Benzene & $\mathrm{CCl} 4$ & 329.30 & 43.90 & 0.8170 & 0.0250 & 0.1580 & 0.5680 & 0.0330 & 0.3990 \\
\hline 204 & Acetonitrile & Benzene & $\mathrm{CCl} 4$ & 297.50 & 39.66 & 0.8290 & 0.0750 & 0.0960 & 0.6150 & 0.1120 & 0.2730 \\
\hline 205 & Acetonitrile & Benzene & $\mathrm{CCl} 4$ & 259.90 & 34.65 & 0.8350 & 0.1540 & 0.0110 & 0.7030 & 0.2630 & 0.0340 \\
\hline 206 & Acetonitrile & Benzene & $\mathrm{CCl} 4$ & 316.00 & 42.13 & 0.8560 & 0.0200 & 0.1240 & 0.5960 & 0.0290 & 0.3750 \\
\hline 207 & Acetonitrile & Benzene & $\mathrm{CCl} 4$ & 304.20 & 40.56 & 0.8780 & 0.0190 & 0.1030 & 0.6220 & 0.0280 & 0.3500 \\
\hline 208 & Acetonitrile & Benzene & $\mathrm{CCl} 4$ & 291.60 & 38.88 & 0.9030 & 0.0150 & 0.0820 & 0.6730 & 0.0270 & 0.3000 \\
\hline 209 & Acetonitrile & Benzene & & 239.70 & 31.96 & 0.0455 & 0.9545 & 0.0000 & 0.1056 & 0.8944 & 0.0000 \\
\hline 210 & Acetonitrile & Benzene & & 251.67 & 33.55 & 0.0940 & 0.9060 & 0.0000 & 0.1818 & 0.8182 & 0.0000 \\
\hline 211 & Acetonitrile & Benzene & & 264.66 & 35.29 & 0.1829 & 0.8171 & 0.0000 & 0.2783 & 0.7217 & 0.0000 \\
\hline
\end{tabular}




\begin{tabular}{|c|c|c|c|c|c|c|c|c|c|c|c|}
\hline 212 & Acetonitrile & Benzene & & 273.45 & 36.46 & 0.2909 & 0.7091 & 0.0000 & 0.3607 & 0.6393 & 0.0000 \\
\hline 213 & Acetonitrile & Benzene & & 277.49 & 37.00 & 0.3980 & 0.6020 & 0.0000 & 0.4274 & 0.5726 & 0.0000 \\
\hline 214 & Acetonitrile & Benzene & & 278.03 & 37.07 & 0.5069 & 0.4931 & 0.0000 & 0.4885 & 0.5115 & 0.0000 \\
\hline 215 & Acetonitrile & Benzene & & 277.36 & 36.98 & 0.5458 & 0.4542 & 0.0000 & 0.5098 & 0.4902 & 0.0000 \\
\hline 216 & Acetonitrile & Benzene & & 275.86 & 36.78 & 0.5946 & 0.4054 & 0.0000 & 0.5375 & 0.4625 & 0.0000 \\
\hline 217 & Acetonitrile & Benzene & & 268.46 & 35.79 & 0.7206 & 0.2794 & 0.0000 & 0.6157 & 0.3843 & 0.0000 \\
\hline 218 & Acetonitrile & Benzene & & 257.81 & 34.37 & 0.8145 & 0.1855 & 0.0000 & 0.6913 & 0.3087 & 0.0000 \\
\hline 219 & Acetonitrile & Benzene & & 242.50 & 32.33 & 0.8972 & 0.1028 & 0.0000 & 0.7869 & 0.2131 & 0.0000 \\
\hline 220 & Acetonitrile & Benzene & & 225.30 & 30.04 & 0.9573 & 0.0427 & 0.0000 & 0.8916 & 0.1084 & 0.0000 \\
\hline 221 & Acetonitrile & & $\mathrm{CCl} 4$ & 314.43 & 41.92 & 0.0391 & 0.0000 & 0.9609 & 0.1999 & 0.0000 & 0.8001 \\
\hline 222 & Acetonitrile & & $\mathrm{CCl} 4$ & 346.97 & 46.26 & 0.1041 & 0.0000 & 0.8959 & 0.2996 & 0.0000 & 0.7004 \\
\hline 223 & Acetonitrile & & $\mathrm{CCl} 4$ & 362.78 & 48.37 & 0.1931 & 0.0000 & 0.8069 & 0.3530 & 0.0000 & 0.6470 \\
\hline 224 & Acetonitrile & & $\mathrm{CCl} 4$ & 368.95 & 49.19 & 0.2836 & 0.0000 & 0.7164 & 0.3819 & 0.0000 & 0.6181 \\
\hline 225 & Acetonitrile & & $\mathrm{CCl} 4$ & 371.09 & 49.47 & 0.3951 & 0.0000 & 0.6049 & 0.4064 & 0.0000 & 0.5936 \\
\hline 226 & Acetonitrile & & $\mathrm{CCl} 4$ & 370.23 & 49.36 & 0.4940 & 0.0000 & 0.5060 & 0.4260 & 0.0000 & 0.5740 \\
\hline 227 & Acetonitrile & & $\mathrm{CCl} 4$ & 369.62 & 49.28 & 0.5210 & 0.0000 & 0.4790 & 0.4316 & 0.0000 & 0.5684 \\
\hline 228 & Acetonitrile & & $\mathrm{CCl} 4$ & 368.82 & 49.17 & 0.5433 & 0.0000 & 0.4567 & 0.4367 & 0.0000 & 0.5633 \\
\hline 229 & Acetonitrile & & $\mathrm{CCl} 4$ & 364.57 & 48.61 & 0.6248 & 0.0000 & 0.3752 & 0.4571 & 0.0000 & 0.5429 \\
\hline 230 & Acetonitrile & & $\mathrm{CCl} 4$ & 355.85 & 47.44 & 0.7113 & 0.0000 & 0.2887 & 0.4871 & 0.0000 & 0.5129 \\
\hline 231 & Acetonitrile & & $\mathrm{CCl} 4$ & 335.99 & 44.79 & 0.8086 & 0.0000 & 0.1914 & 0.5397 & 0.0000 & 0.4603 \\
\hline 232 & Acetonitrile & & $\mathrm{CCl} 4$ & 291.58 & 38.87 & 0.9108 & 0.0000 & 0.0892 & 0.6691 & 0.0000 & 0.3309 \\
\hline 233 & Acetonitrile & & $\mathrm{CCl} 4$ & 247.96 & 33.06 & 0.9653 & 0.0000 & 0.0347 & 0.8199 & 0.0000 & 0.1801 \\
\hline 234 & & Benzene & $\mathrm{CCl} 4$ & 255.43 & 34.05 & 0.0000 & 0.1000 & 0.9000 & 0.0000 & 0.1012 & 0.8988 \\
\hline 235 & & Benzene & $\mathrm{CCl} 4$ & 254.65 & 33.95 & 0.0000 & 0.2500 & 0.7500 & 0.0000 & 0.2399 & 0.7601 \\
\hline 236 & & Benzene & $\mathrm{CCl} 4$ & 251.70 & 33.56 & 0.0000 & 0.4000 & 0.6000 & 0.0000 & 0.3736 & 0.6264 \\
\hline 237 & & Benzene & $\mathrm{CCl} 4$ & 248.73 & 33.16 & 0.0000 & 0.5000 & 0.5000 & 0.0000 & 0.4642 & 0.5358 \\
\hline 238 & & Benzene & $\mathrm{CCl} 4$ & 245.01 & 32.66 & 0.0000 & 0.6000 & 0.4000 & 0.0000 & 0.5582 & 0.4418 \\
\hline
\end{tabular}




\begin{tabular}{|c|c|c|c|c|c|c|c|c|c|c|c|}
\hline 239 & & Benzene & $\mathrm{CCl} 4$ & 240.59 & 32.08 & 0.0000 & 0.7000 & 0.3000 & 0.0000 & 0.6572 & 0.3428 \\
\hline 240 & & Benzene & $\mathrm{CCl} 4$ & 238.13 & 31.75 & 0.0000 & 0.7500 & 0.2500 & 0.0000 & 0.7091 & 0.2909 \\
\hline 241 & & Benzene & $\mathrm{CCl} 4$ & 229.83 & 30.64 & 0.0000 & 0.9000 & 0.1000 & 0.0000 & 0.8767 & 0.1233 \\
\hline 242 & Benzene & Cyclohexane & Ethanol & 453.50 & 60.46 & 0.0840 & 0.4580 & 0.4580 & 0.0930 & 0.5080 & 0.3990 \\
\hline 243 & Benzene & Cyclohexane & Ethanol & 425.60 & 56.74 & 0.1140 & 0.2660 & 0.6200 & 0.1510 & 0.4400 & 0.4090 \\
\hline 244 & Benzene & Cyclohexane & Ethanol & 450.50 & 60.06 & 0.1140 & 0.6200 & 0.2660 & 0.1040 & 0.5110 & 0.3850 \\
\hline 245 & Benzene & Cyclohexane & Ethanol & 403.00 & 53.73 & 0.1345 & 0.1345 & 0.7310 & 0.2260 & 0.3220 & 0.4520 \\
\hline 246 & Benzene & Cyclohexane & Ethanol & 422.00 & 56.26 & 0.1345 & 0.7310 & 0.1345 & 0.1190 & 0.5280 & 0.3530 \\
\hline 247 & Benzene & Cyclohexane & Ethanol & 451.00 & 60.13 & 0.1760 & 0.4120 & 0.4120 & 0.1860 & 0.4430 & 0.3710 \\
\hline 248 & Benzene & Cyclohexane & Ethanol & 425.30 & 56.70 & 0.2310 & 0.2310 & 0.5380 & 0.2630 & 0.3620 & 0.3750 \\
\hline 249 & Benzene & Cyclohexane & Ethanol & 434.00 & 57.86 & 0.2310 & 0.5380 & 0.2310 & 0.1950 & 0.4500 & 0.3550 \\
\hline 250 & Benzene & Cyclohexane & Ethanol & 408.00 & 54.40 & 0.2660 & 0.1140 & 0.6200 & 0.3630 & 0.2240 & 0.4130 \\
\hline 251 & Benzene & Cyclohexane & Ethanol & 417.50 & 55.66 & 0.2660 & 0.6200 & 0.1140 & 0.2200 & 0.4740 & 0.3060 \\
\hline 252 & Benzene & Cyclohexane & Ethanol & 429.50 & 57.26 & 0.3330 & 0.3330 & 0.3340 & 0.2950 & 0.3540 & 0.3510 \\
\hline 253 & Benzene & Cyclohexane & Ethanol & 417.00 & 55.60 & 0.4120 & 0.1760 & 0.4120 & 0.4020 & 0.2290 & 0.3690 \\
\hline 254 & Benzene & Cyclohexane & Ethanol & 421.00 & 56.13 & 0.4120 & 0.4120 & 0.1760 & 0.3190 & 0.3620 & 0.3190 \\
\hline 255 & Benzene & Cyclohexane & Ethanol & 404.50 & 53.93 & 0.4580 & 0.0840 & 0.4580 & 0.4880 & 0.1290 & 0.3830 \\
\hline 256 & Benzene & Cyclohexane & Ethanol & 399.50 & 53.26 & 0.4580 & 0.4580 & 0.0840 & 0.3590 & 0.3840 & 0.2570 \\
\hline 257 & Benzene & Cyclohexane & Ethanol & 418.60 & 55.81 & 0.5380 & 0.2310 & 0.2310 & 0.4260 & 0.2410 & 0.3330 \\
\hline 258 & Benzene & Cyclohexane & Ethanol & 403.00 & 53.73 & 0.6200 & 0.1140 & 0.2660 & 0.5200 & 0.1340 & 0.3460 \\
\hline 259 & Benzene & Cyclohexane & Ethanol & 403.00 & 53.73 & 0.6200 & 0.2660 & 0.1140 & 0.4640 & 0.2550 & 0.2810 \\
\hline 260 & Benzene & Cyclohexane & Ethanol & 397.50 & 53.00 & 0.7310 & 0.1345 & 0.1345 & 0.5560 & 0.1420 & 0.3020 \\
\hline 261 & Benzene & & Ethanol & 314.70 & 41.96 & 0.9750 & 0.0000 & 0.0250 & 0.8350 & 0.0000 & 0.1650 \\
\hline 262 & Benzene & & Ethanol & 358.70 & 47.82 & 0.9110 & 0.0000 & 0.0890 & 0.7000 & 0.0000 & 0.3000 \\
\hline 263 & Benzene & & Ethanol & 378.30 & 50.44 & 0.7940 & 0.0000 & 0.2060 & 0.6400 & 0.0000 & 0.3600 \\
\hline 264 & Benzene & & Ethanol & 384.60 & 51.28 & 0.6150 & 0.0000 & 0.3850 & 0.6080 & 0.0000 & 0.3920 \\
\hline 265 & Benzene & & Ethanol & 383.20 & 51.09 & 0.5140 & 0.0000 & 0.4860 & 0.5890 & 0.0000 & 0.4110 \\
\hline
\end{tabular}




\begin{tabular}{|c|c|c|c|c|c|c|c|c|c|c|c|}
\hline 266 & Benzene & & Ethanol & 378.10 & 50.41 & 0.4140 & 0.0000 & 0.5860 & 0.5660 & 0.0000 & 0.4340 \\
\hline 267 & Benzene & & Ethanol & 366.90 & 48.92 & 0.3060 & 0.0000 & 0.6940 & 0.5300 & 0.0000 & 0.4700 \\
\hline 268 & Benzene & & Ethanol & 344.40 & 45.92 & 0.2100 & 0.0000 & 0.7900 & 0.4740 & 0.0000 & 0.5260 \\
\hline 269 & Benzene & & Ethanol & 316.80 & 42.24 & 0.1340 & 0.0000 & 0.8660 & 0.3900 & 0.0000 & 0.6100 \\
\hline 270 & Benzene & & Ethanol & 276.80 & 36.90 & 0.0640 & 0.0000 & 0.9360 & 0.2550 & 0.0000 & 0.7450 \\
\hline 271 & Benzene & & Ethanol & 239.60 & 31.94 & 0.0160 & 0.0000 & 0.9840 & 0.0910 & 0.0000 & 0.9090 \\
\hline 272 & Benzene & Cyclohexane & & 284.03 & 37.87 & 0.1000 & 0.9000 & 0.0000 & 0.1342 & 0.8658 & 0.0000 \\
\hline 273 & Benzene & Cyclohexane & & 292.57 & 39.01 & 0.2000 & 0.8000 & 0.0000 & 0.2432 & 0.7568 & 0.0000 \\
\hline 274 & Benzene & Cyclohexane & & 298.29 & 39.77 & 0.3000 & 0.7000 & 0.0000 & 0.3371 & 0.6629 & 0.0000 \\
\hline 275 & Benzene & Cyclohexane & & 301.64 & 40.22 & 0.4000 & 0.6000 & 0.0000 & 0.4220 & 0.5780 & 0.0000 \\
\hline 276 & Benzene & Cyclohexane & & 302.89 & 40.38 & 0.5000 & 0.5000 & 0.0000 & 0.5024 & 0.4976 & 0.0000 \\
\hline 277 & Benzene & Cyclohexane & & 302.10 & 40.28 & 0.6000 & 0.4000 & 0.0000 & 0.5821 & 0.4179 & 0.0000 \\
\hline 278 & Benzene & Cyclohexane & & 299.15 & 39.88 & 0.7000 & 0.3000 & 0.0000 & 0.6649 & 0.3351 & 0.0000 \\
\hline 279 & Benzene & Cyclohexane & & 293.67 & 39.15 & 0.8000 & 0.2000 & 0.0000 & 0.7560 & 0.2440 & 0.0000 \\
\hline 280 & & Cyclohexane & Ethanol & 408.42 & 54.45 & 0.0000 & 0.9094 & 0.0906 & 0.0000 & 0.6419 & 0.3581 \\
\hline 281 & & Cyclohexane & Ethanol & 423.42 & 56.45 & 0.0000 & 0.7555 & 0.2445 & 0.0000 & 0.6061 & 0.3939 \\
\hline 282 & & Cyclohexane & Ethanol & 425.81 & 56.77 & 0.0000 & 0.6148 & 0.3852 & 0.0000 & 0.5942 & 0.4058 \\
\hline 283 & & Cyclohexane & Ethanol & 425.12 & 56.68 & 0.0000 & 0.5072 & 0.4928 & 0.0000 & 0.5844 & 0.4156 \\
\hline 284 & & Cyclohexane & Ethanol & 421.89 & 56.25 & 0.0000 & 0.3903 & 0.6097 & 0.0000 & 0.5690 & 0.4310 \\
\hline 285 & & Cyclohexane & Ethanol & 405.91 & 54.12 & 0.0000 & 0.2413 & 0.7587 & 0.0000 & 0.5293 & 0.4707 \\
\hline 286 & & Cyclohexane & Ethanol & 340.29 & 45.37 & 0.0000 & 0.0886 & 0.9114 & 0.0000 & 0.3956 & 0.6044 \\
\hline 287 & $\mathrm{CH} 2 \mathrm{Cl} 2$ & $\mathrm{CHCl} 3$ & $\mathrm{CCl} 4$ & 292.50 & 39.00 & 0.0240 & 0.0460 & 0.9300 & 0.1050 & 0.0750 & 0.8200 \\
\hline 288 & $\mathrm{CH} 2 \mathrm{Cl} 2$ & $\mathrm{CHCl} 3$ & $\mathrm{CCl} 4$ & 333.30 & 44.44 & 0.0660 & 0.0400 & 0.8940 & 0.2510 & 0.0520 & 0.6970 \\
\hline 289 & $\mathrm{CH} 2 \mathrm{Cl} 2$ & $\mathrm{CHCl} 3$ & $\mathrm{CCl} 4$ & 489.40 & 65.25 & 0.2570 & 0.0160 & 0.7270 & 0.5940 & 0.0140 & 0.3920 \\
\hline 290 & $\mathrm{CH} 2 \mathrm{Cl} 2$ & $\mathrm{CHCl} 3$ & $\mathrm{CCl} 4$ & 608.70 & 81.15 & 0.4210 & 0.0160 & 0.5630 & 0.7380 & 0.0120 & 0.2500 \\
\hline 291 & $\mathrm{CH} 2 \mathrm{Cl} 2$ & $\mathrm{CHCl} 3$ & $\mathrm{CCl} 4$ & 313.80 & 41.84 & 0.0330 & 0.0950 & 0.8720 & 0.1440 & 0.1420 & 0.7140 \\
\hline 292 & $\mathrm{CH} 2 \mathrm{Cl} 2$ & $\mathrm{CHCl} 3$ & $\mathrm{CCl} 4$ & 390.50 & 52.06 & 0.1230 & 0.0760 & 0.8010 & 0.3820 & 0.0880 & 0.5300 \\
\hline
\end{tabular}




\begin{tabular}{|c|c|c|c|c|c|c|c|c|c|c|c|}
\hline 293 & $\mathrm{CH} 2 \mathrm{Cl} 2$ & $\mathrm{CHCl} 3$ & $\mathrm{CCl} 4$ & 524.60 & 69.94 & 0.3010 & 0.0520 & 0.6470 & 0.6250 & 0.0430 & 0.3320 \\
\hline 294 & $\mathrm{CH} 2 \mathrm{Cl} 2$ & $\mathrm{CHCl} 3$ & $\mathrm{CCl} 4$ & 684.90 & 91.31 & 0.5670 & 0.0270 & 0.4060 & 0.8020 & 0.0170 & 0.1810 \\
\hline 295 & $\mathrm{CH} 2 \mathrm{Cl} 2$ & $\mathrm{CHCl} 3$ & $\mathrm{CCl} 4$ & 854.80 & 113.96 & 0.8820 & 0.0100 & 0.1080 & 0.9450 & 0.0060 & 0.0490 \\
\hline 296 & $\mathrm{CH} 2 \mathrm{Cl} 2$ & $\mathrm{CHCl} 3$ & $\mathrm{CCl} 4$ & 331.40 & 44.18 & 0.0310 & 0.2100 & 0.7590 & 0.1230 & 0.2780 & 0.5990 \\
\hline 297 & $\mathrm{CH} 2 \mathrm{Cl} 2$ & $\mathrm{CHCl} 3$ & $\mathrm{CCl} 4$ & 392.60 & 52.34 & 0.1010 & 0.1840 & 0.7150 & 0.2900 & 0.2140 & 0.4960 \\
\hline 298 & $\mathrm{CH} 2 \mathrm{Cl} 2$ & $\mathrm{CHCl} 3$ & $\mathrm{CCl} 4$ & 465.30 & 62.03 & 0.2060 & 0.1610 & 0.6330 & 0.4920 & 0.1480 & 0.3600 \\
\hline 299 & $\mathrm{CH} 2 \mathrm{Cl} 2$ & $\mathrm{CHCl} 3$ & $\mathrm{CCl} 4$ & 576.30 & 76.83 & 0.3850 & 0.1160 & 0.4990 & 0.6710 & 0.0860 & 0.2430 \\
\hline 300 & $\mathrm{CH} 2 \mathrm{Cl} 2$ & $\mathrm{CHCl} 3$ & $\mathrm{CCl} 4$ & 646.70 & 86.22 & 0.5010 & 0.0860 & 0.4130 & 0.7540 & 0.0610 & 0.1850 \\
\hline 301 & $\mathrm{CH} 2 \mathrm{Cl} 2$ & $\mathrm{CHCl} 3$ & $\mathrm{CCl} 4$ & 762.30 & 101.63 & 0.7070 & 0.0470 & 0.2460 & 0.8690 & 0.0300 & 0.1010 \\
\hline 302 & $\mathrm{CH} 2 \mathrm{Cl} 2$ & $\mathrm{CHCl} 3$ & $\mathrm{CCl} 4$ & 811.90 & 108.24 & 0.8000 & 0.0330 & 0.1670 & 0.9050 & 0.0270 & 0.0680 \\
\hline 303 & $\mathrm{CH} 2 \mathrm{Cl} 2$ & $\mathrm{CHCl} 3$ & $\mathrm{CCl} 4$ & 445.20 & 59.36 & 0.1470 & 0.2520 & 0.6010 & 0.3860 & 0.2550 & 0.3590 \\
\hline 304 & $\mathrm{CH} 2 \mathrm{Cl} 2$ & $\mathrm{CHCl} 3$ & $\mathrm{CCl} 4$ & 512.70 & 68.35 & 0.2590 & 0.2190 & 0.5220 & 0.5430 & 0.1830 & 0.2740 \\
\hline 305 & $\mathrm{CH} 2 \mathrm{Cl} 2$ & $\mathrm{CHCl} 3$ & $\mathrm{CCl} 4$ & 611.90 & 81.58 & 0.4280 & 0.1600 & 0.4120 & 0.6940 & 0.1130 & 0.1930 \\
\hline 306 & $\mathrm{CH} 2 \mathrm{Cl} 2$ & $\mathrm{CHCl} 3$ & $\mathrm{CCl} 4$ & 711.60 & 94.87 & 0.6120 & 0.1030 & 0.2850 & 0.8080 & 0.0660 & 0.1260 \\
\hline 307 & $\mathrm{CH} 2 \mathrm{Cl} 2$ & $\mathrm{CHCl} 3$ & $\mathrm{CCl} 4$ & 782.30 & 104.30 & 0.7510 & 0.0640 & 0.1850 & 0.8800 & 0.0400 & 0.0800 \\
\hline 308 & $\mathrm{CH} 2 \mathrm{Cl} 2$ & $\mathrm{CHCl} 3$ & $\mathrm{CCl} 4$ & 830.70 & 110.75 & 0.8380 & 0.0480 & 0.1140 & 0.9120 & 0.0340 & 0.0540 \\
\hline 309 & $\mathrm{CH} 2 \mathrm{Cl} 2$ & $\mathrm{CHCl} 3$ & $\mathrm{CCl} 4$ & 366.70 & 48.89 & 0.0300 & 0.3920 & 0.5780 & 0.0970 & 0.4900 & 0.4130 \\
\hline 310 & $\mathrm{CH} 2 \mathrm{Cl} 2$ & $\mathrm{CHCl} 3$ & $\mathrm{CCl} 4$ & 429.00 & 57.20 & 0.1190 & 0.3520 & 0.5290 & 0.2920 & 0.3720 & 0.3360 \\
\hline 311 & $\mathrm{CH} 2 \mathrm{Cl} 2$ & $\mathrm{CHCl} 3$ & $\mathrm{CCl} 4$ & 526.20 & 70.15 & 0.2710 & 0.2930 & 0.4360 & 0.5130 & 0.2490 & 0.2380 \\
\hline 312 & $\mathrm{CH} 2 \mathrm{Cl} 2$ & $\mathrm{CHCl} 3$ & $\mathrm{CCl} 4$ & 623.10 & 83.07 & 0.4390 & 0.2100 & 0.3510 & 0.6800 & 0.1460 & 0.1740 \\
\hline 313 & $\mathrm{CH} 2 \mathrm{Cl} 2$ & $\mathrm{CHCl} 3$ & $\mathrm{CCl} 4$ & 715.70 & 95.42 & 0.6170 & 0.1430 & 0.2400 & 0.8080 & 0.0860 & 0.1060 \\
\hline 314 & $\mathrm{CH} 2 \mathrm{Cl} 2$ & $\mathrm{CHCl} 3$ & $\mathrm{CCl} 4$ & 787.60 & 105.00 & 0.7620 & 0.0880 & 0.1500 & 0.8790 & 0.0510 & 0.0700 \\
\hline 315 & $\mathrm{CH} 2 \mathrm{Cl} 2$ & $\mathrm{CHCl} 3$ & $\mathrm{CCl} 4$ & 824.30 & 109.90 & 0.8330 & 0.0620 & 0.1050 & 0.9070 & 0.0430 & 0.0500 \\
\hline 316 & $\mathrm{CH} 2 \mathrm{Cl} 2$ & $\mathrm{CHCl} 3$ & $\mathrm{CCl} 4$ & 381.10 & 50.81 & 0.0270 & 0.4930 & 0.4800 & 0.0830 & 0.5770 & 0.3400 \\
\hline 317 & $\mathrm{CH} 2 \mathrm{Cl} 2$ & $\mathrm{CHCl} 3$ & $\mathrm{CCl} 4$ & 410.90 & 54.78 & 0.0740 & 0.4620 & 0.4640 & 0.1920 & 0.5070 & 0.3010 \\
\hline 318 & $\mathrm{CH} 2 \mathrm{Cl} 2$ & $\mathrm{CHCl} 3$ & $\mathrm{CCl} 4$ & 479.60 & 63.94 & 0.1810 & 0.4030 & 0.4160 & 0.3900 & 0.3730 & 0.2370 \\
\hline 319 & $\mathrm{CH} 2 \mathrm{Cl} 2$ & $\mathrm{CHCl} 3$ & $\mathrm{CCl} 4$ & 577.10 & 76.94 & 0.3480 & 0.3250 & 0.3270 & 0.5800 & 0.2420 & 0.1780 \\
\hline
\end{tabular}




\begin{tabular}{|c|c|c|c|c|c|c|c|c|c|c|c|}
\hline 320 & $\mathrm{CH} 2 \mathrm{Cl} 2$ & $\mathrm{CHCl} 3$ & $\mathrm{CCl} 4$ & 696.30 & 92.83 & 0.5690 & 0.2140 & 0.2170 & 0.7720 & 0.1330 & 0.0950 \\
\hline 321 & $\mathrm{CH} 2 \mathrm{Cl} 2$ & $\mathrm{CHCl} 3$ & $\mathrm{CCl} 4$ & 766.80 & 102.23 & 0.7110 & 0.1490 & 0.1400 & 0.8550 & 0.0840 & 0.0610 \\
\hline 322 & $\mathrm{CH} 2 \mathrm{Cl} 2$ & $\mathrm{CHCl} 3$ & $\mathrm{CCl} 4$ & 817.30 & 108.96 & 0.8150 & 0.0870 & 0.0980 & 0.9120 & 0.0480 & 0.0400 \\
\hline 323 & $\mathrm{CH} 2 \mathrm{Cl} 2$ & $\mathrm{CHCl} 3$ & $\mathrm{CCl} 4$ & 394.60 & 52.61 & 0.0250 & 0.5910 & 0.3840 & 0.0800 & 0.6600 & 0.2600 \\
\hline 324 & $\mathrm{CH} 2 \mathrm{Cl} 2$ & $\mathrm{CHCl} 3$ & $\mathrm{CCl} 4$ & 432.10 & 57.61 & 0.0910 & 0.5410 & 0.3680 & 0.2150 & 0.5540 & 0.2310 \\
\hline 325 & $\mathrm{CH} 2 \mathrm{Cl} 2$ & $\mathrm{CHCl} 3$ & $\mathrm{CCl} 4$ & 526.60 & 70.21 & 0.2520 & 0.4470 & 0.3010 & 0.4770 & 0.3670 & 0.1560 \\
\hline 326 & $\mathrm{CH} 2 \mathrm{Cl} 2$ & $\mathrm{CHCl} 3$ & $\mathrm{CCl} 4$ & 607.00 & 80.93 & 0.4010 & 0.3560 & 0.2430 & 0.6140 & 0.2600 & 0.1260 \\
\hline 327 & $\mathrm{CH} 2 \mathrm{Cl} 2$ & $\mathrm{CHCl} 3$ & $\mathrm{CCl} 4$ & 701.70 & 93.55 & 0.5870 & 0.2410 & 0.1720 & 0.7700 & 0.1470 & 0.0830 \\
\hline 328 & $\mathrm{CH} 2 \mathrm{Cl} 2$ & $\mathrm{CHCl} 3$ & $\mathrm{CCl} 4$ & 777.80 & 103.70 & 0.7430 & 0.1500 & 0.1070 & 0.8680 & 0.0840 & 0.0480 \\
\hline 329 & $\mathrm{CH} 2 \mathrm{Cl} 2$ & $\mathrm{CHCl} 3$ & $\mathrm{CCl} 4$ & 407.50 & 54.33 & 0.0220 & 0.6780 & 0.3000 & 0.0680 & 0.7290 & 0.2030 \\
\hline 330 & $\mathrm{CH} 2 \mathrm{Cl} 2$ & $\mathrm{CHCl} 3$ & $\mathrm{CCl} 4$ & 517.90 & 69.05 & 0.2140 & 0.5460 & 0.2400 & 0.4100 & 0.4620 & 0.1280 \\
\hline 331 & $\mathrm{CH} 2 \mathrm{Cl} 2$ & $\mathrm{CHCl} 3$ & $\mathrm{CCl} 4$ & 598.00 & 79.73 & 0.3760 & 0.4330 & 0.1910 & 0.5930 & 0.3130 & 0.0940 \\
\hline 332 & $\mathrm{CH} 2 \mathrm{Cl} 2$ & $\mathrm{CHCl} 3$ & $\mathrm{CCl} 4$ & 696.30 & 92.83 & 0.5600 & 0.3050 & 0.1350 & 0.7480 & 0.1870 & 0.0650 \\
\hline 333 & $\mathrm{CH} 2 \mathrm{Cl} 2$ & $\mathrm{CHCl} 3$ & $\mathrm{CCl} 4$ & 800.40 & 106.71 & 0.7870 & 0.1420 & 0.0710 & 0.8830 & 0.0860 & 0.0310 \\
\hline 334 & $\mathrm{CH} 2 \mathrm{Cl} 2$ & $\mathrm{CHCl} 3$ & $\mathrm{CCl} 4$ & 837.10 & 111.60 & 0.8540 & 0.0990 & 0.0470 & 0.9180 & 0.0610 & 0.0210 \\
\hline 335 & $\mathrm{CH} 2 \mathrm{Cl} 2$ & $\mathrm{CHCl} 3$ & $\mathrm{CCl} 4$ & 420.50 & 56.06 & 0.0180 & 0.7800 & 0.2020 & 0.0500 & 0.8240 & 0.1260 \\
\hline 336 & $\mathrm{CH} 2 \mathrm{Cl} 2$ & $\mathrm{CHCl} 3$ & $\mathrm{CCl} 4$ & 482.40 & 64.31 & 0.1340 & 0.6890 & 0.1770 & 0.2790 & 0.6240 & 0.0970 \\
\hline 337 & $\mathrm{CH} 2 \mathrm{Cl} 2$ & $\mathrm{CHCl} 3$ & $\mathrm{CCl} 4$ & 589.60 & 78.61 & 0.3440 & 0.5290 & 0.1270 & 0.5460 & 0.3910 & 0.0630 \\
\hline 338 & $\mathrm{CH} 2 \mathrm{Cl} 2$ & $\mathrm{CHCl} 3$ & $\mathrm{CCl} 4$ & 718.80 & 95.83 & 0.6080 & 0.3200 & 0.0720 & 0.7780 & 0.1900 & 0.0320 \\
\hline 339 & $\mathrm{CH} 2 \mathrm{Cl} 2$ & $\mathrm{CHCl} 3$ & $\mathrm{CCl} 4$ & 788.50 & 105.12 & 0.7570 & 0.1980 & 0.0450 & 0.8740 & 0.1070 & 0.0190 \\
\hline 340 & $\mathrm{CH} 2 \mathrm{Cl} 2$ & $\mathrm{CHCl} 3$ & $\mathrm{CCl} 4$ & 814.70 & 108.62 & 0.8150 & 0.1460 & 0.0390 & 0.8970 & 0.0840 & 0.0190 \\
\hline 341 & $\mathrm{CH} 2 \mathrm{Cl} 2$ & $\mathrm{CHCl} 3$ & $\mathrm{CCl} 4$ & 430.40 & 57.38 & 0.0170 & 0.8820 & 0.1010 & 0.0540 & 0.8860 & 0.0600 \\
\hline 342 & $\mathrm{CH} 2 \mathrm{Cl} 2$ & $\mathrm{CHCl} 3$ & $\mathrm{CCl} 4$ & 529.00 & 70.53 & 0.1990 & 0.7250 & 0.0760 & 0.3740 & 0.5860 & 0.0400 \\
\hline 343 & $\mathrm{CH} 2 \mathrm{Cl} 2$ & $\mathrm{CHCl} 3$ & $\mathrm{CCl} 4$ & 695.50 & 92.73 & 0.5480 & 0.4090 & 0.0430 & 0.7280 & 0.2510 & 0.0210 \\
\hline 344 & $\mathrm{CH} 2 \mathrm{Cl} 2$ & $\mathrm{CHCl} 3$ & $\mathrm{CCl} 4$ & 780.60 & 104.07 & 0.7280 & 0.2440 & 0.0280 & 0.8370 & 0.1490 & 0.0140 \\
\hline 345 & $\mathrm{CH} 2 \mathrm{Cl} 2$ & $\mathrm{CHCl} 3$ & & 447.50 & 59.66 & 0.0250 & 0.9750 & 0.0000 & 0.0570 & 0.9430 & 0.0000 \\
\hline 346 & $\mathrm{CH} 2 \mathrm{Cl} 2$ & $\mathrm{CHCl} 3$ & & 469.70 & 62.62 & 0.0500 & 0.9500 & 0.0000 & 0.1060 & 0.8940 & 0.0000 \\
\hline
\end{tabular}




\begin{tabular}{|c|c|c|c|c|c|c|c|c|c|c|c|}
\hline 347 & $\mathrm{CH} 2 \mathrm{Cl} 2$ & $\mathrm{CHCl} 3$ & & 485.30 & 64.70 & 0.1000 & 0.9000 & 0.0000 & 0.1960 & 0.8040 & 0.0000 \\
\hline 348 & $\mathrm{CH} 2 \mathrm{Cl} 2$ & $\mathrm{CHCl} 3$ & & 509.20 & 67.89 & 0.1500 & 0.8500 & 0.0000 & 0.2760 & 0.7240 & 0.0000 \\
\hline 349 & $\mathrm{CH} 2 \mathrm{Cl} 2$ & $\mathrm{CHCl} 3$ & & 532.50 & 70.99 & 0.2000 & 0.8000 & 0.0000 & 0.3490 & 0.6510 & 0.0000 \\
\hline 350 & $\mathrm{CH} 2 \mathrm{Cl} 2$ & $\mathrm{CHCl} 3$ & & 556.00 & 74.13 & 0.2500 & 0.7500 & 0.0000 & 0.4160 & 0.5840 & 0.0000 \\
\hline 351 & $\mathrm{CH} 2 \mathrm{Cl} 2$ & $\mathrm{CHCl} 3$ & & 580.30 & 77.37 & 0.3000 & 0.7000 & 0.0000 & 0.4780 & 0.5220 & 0.0000 \\
\hline 352 & $\mathrm{CH} 2 \mathrm{Cl} 2$ & $\mathrm{CHCl} 3$ & & 603.60 & 80.47 & 0.3500 & 0.6500 & 0.0000 & 0.5320 & 0.4680 & 0.0000 \\
\hline 353 & $\mathrm{CH} 2 \mathrm{Cl} 2$ & $\mathrm{CHCl} 3$ & & 626.70 & 83.55 & 0.4000 & 0.6000 & 0.0000 & 0.5840 & 0.4160 & 0.0000 \\
\hline 354 & $\mathrm{CH} 2 \mathrm{Cl} 2$ & $\mathrm{CHCl} 3$ & & 650.00 & 86.66 & 0.4500 & 0.5500 & 0.0000 & 0.6340 & 0.3660 & 0.0000 \\
\hline 355 & $\mathrm{CH} 2 \mathrm{Cl} 2$ & $\mathrm{CHCl} 3$ & & 672.80 & 89.70 & 0.5000 & 0.5000 & 0.0000 & 0.6760 & 0.3240 & 0.0000 \\
\hline 356 & $\mathrm{CH} 2 \mathrm{Cl} 2$ & $\mathrm{CHCl} 3$ & & 696.00 & 92.79 & 0.5500 & 0.4500 & 0.0000 & 0.7180 & 0.2820 & 0.0000 \\
\hline 357 & $\mathrm{CH} 2 \mathrm{Cl} 2$ & $\mathrm{CHCl} 3$ & & 719.60 & 95.94 & 0.6000 & 0.4000 & 0.0000 & 0.7570 & 0.2430 & 0.0000 \\
\hline 358 & $\mathrm{CH} 2 \mathrm{Cl} 2$ & $\mathrm{CHCl} 3$ & & 742.90 & 99.05 & 0.6500 & 0.3500 & 0.0000 & 0.7940 & 0.2060 & 0.0000 \\
\hline 359 & $\mathrm{CH} 2 \mathrm{Cl} 2$ & $\mathrm{CHCl} 3$ & & 766.70 & 102.22 & 0.7000 & 0.3000 & 0.0000 & 0.8280 & 0.1720 & 0.0000 \\
\hline 360 & $\mathrm{CH} 2 \mathrm{Cl} 2$ & $\mathrm{CHCl} 3$ & & 790.70 & 105.42 & 0.7500 & 0.2500 & 0.0000 & 0.8600 & 0.1400 & 0.0000 \\
\hline 361 & $\mathrm{CH} 2 \mathrm{Cl} 2$ & $\mathrm{CHCl} 3$ & & 814.60 & 108.60 & 0.8000 & 0.2000 & 0.0000 & 0.8910 & 0.1090 & 0.0000 \\
\hline 362 & $\mathrm{CH} 2 \mathrm{Cl} 2$ & $\mathrm{CHCl} 3$ & & 838.60 & 111.80 & 0.8500 & 0.1500 & 0.0000 & 0.9200 & 0.0800 & 0.0000 \\
\hline 363 & $\mathrm{CH} 2 \mathrm{Cl} 2$ & $\mathrm{CHCl} 3$ & & 862.30 & 114.96 & 0.9000 & 0.1000 & 0.0000 & 0.9470 & 0.0530 & 0.0000 \\
\hline 364 & $\mathrm{CH} 2 \mathrm{Cl} 2$ & $\mathrm{CHCl} 3$ & & 872.00 & 116.26 & 0.9200 & 0.0800 & 0.0000 & 0.9580 & 0.0420 & 0.0000 \\
\hline 365 & $\mathrm{CH} 2 \mathrm{Cl} 2$ & $\mathrm{CHCl} 3$ & & 886.30 & 118.16 & 0.9500 & 0.0500 & 0.0000 & 0.9740 & 0.0260 & 0.0000 \\
\hline 366 & $\mathrm{CH} 2 \mathrm{Cl} 2$ & $\mathrm{CHCl} 3$ & & 896.30 & 119.50 & 0.9700 & 0.0300 & 0.0000 & 0.9840 & 0.0160 & 0.0000 \\
\hline 367 & $\mathrm{CH} 2 \mathrm{Cl} 2$ & & $\mathrm{CCl} 4$ & 285.50 & 38.06 & 0.0250 & 0.0000 & 0.9750 & 0.1170 & 0.0000 & 0.8830 \\
\hline 368 & $\mathrm{CH} 2 \mathrm{Cl} 2$ & & $\mathrm{CCl} 4$ & 310.50 & 41.40 & 0.0500 & 0.0000 & 0.9500 & 0.2110 & 0.0000 & 0.7890 \\
\hline 369 & $\mathrm{CH} 2 \mathrm{Cl} 2$ & & $\mathrm{CCl} 4$ & 358.00 & 47.73 & 0.1000 & 0.0000 & 0.9000 & 0.3520 & 0.0000 & 0.6480 \\
\hline 370 & $\mathrm{CH} 2 \mathrm{Cl} 2$ & & $\mathrm{CCl} 4$ & 404.00 & 53.86 & 0.1500 & 0.0000 & 0.8500 & 0.4540 & 0.0000 & 0.5460 \\
\hline 371 & $\mathrm{CH} 2 \mathrm{Cl} 2$ & & $\mathrm{CCl} 4$ & 444.50 & 59.26 & 0.2000 & 0.0000 & 0.8000 & 0.5340 & 0.0000 & 0.4660 \\
\hline 372 & $\mathrm{CH} 2 \mathrm{Cl} 2$ & & $\mathrm{CCl} 4$ & 481.90 & 64.25 & 0.2500 & 0.0000 & 0.7500 & 0.5940 & 0.0000 & 0.4060 \\
\hline 373 & $\mathrm{CH} 2 \mathrm{Cl} 2$ & & $\mathrm{CCl} 4$ & 518.00 & 69.06 & 0.3000 & 0.0000 & 0.7000 & 0.6430 & 0.0000 & 0.3570 \\
\hline
\end{tabular}




\begin{tabular}{|c|c|c|c|c|c|c|c|c|c|c|c|}
\hline 374 & $\mathrm{CH} 2 \mathrm{Cl} 2$ & & $\mathrm{CCl} 4$ & 552.00 & 73.59 & 0.3500 & 0.0000 & 0.6500 & 0.6850 & 0.0000 & 0.3150 \\
\hline 375 & $\mathrm{CH} 2 \mathrm{Cl} 2$ & & $\mathrm{CCl} 4$ & 585.00 & 77.99 & 0.4000 & 0.0000 & 0.6000 & 0.7220 & 0.0000 & 0.2780 \\
\hline 376 & $\mathrm{CH} 2 \mathrm{Cl} 2$ & & $\mathrm{CCl} 4$ & 616.20 & 82.15 & 0.4500 & 0.0000 & 0.5500 & 0.7530 & 0.0000 & 0.2470 \\
\hline 377 & $\mathrm{CH} 2 \mathrm{Cl} 2$ & & $\mathrm{CCl} 4$ & 644.80 & 85.97 & 0.5000 & 0.0000 & 0.5000 & 0.7800 & 0.0000 & 0.2200 \\
\hline 378 & $\mathrm{CH} 2 \mathrm{Cl} 2$ & & $\mathrm{CCl} 4$ & 673.30 & 89.77 & 0.5500 & 0.0000 & 0.4500 & 0.8070 & 0.0000 & 0.1930 \\
\hline 379 & $\mathrm{CH} 2 \mathrm{Cl} 2$ & & $\mathrm{CCl} 4$ & 700.50 & 93.39 & 0.6000 & 0.0000 & 0.4000 & 0.8300 & 0.0000 & 0.1700 \\
\hline 380 & $\mathrm{CH} 2 \mathrm{Cl} 2$ & & $\mathrm{CCl} 4$ & 726.50 & 96.86 & 0.6500 & 0.0000 & 0.3500 & 0.8510 & 0.0000 & 0.1490 \\
\hline 381 & $\mathrm{CH} 2 \mathrm{Cl} 2$ & & $\mathrm{CCl} 4$ & 752.90 & 100.38 & 0.7000 & 0.0000 & 0.3000 & 0.8720 & 0.0000 & 0.1280 \\
\hline 382 & $\mathrm{CH} 2 \mathrm{Cl} 2$ & & $\mathrm{CCl} 4$ & 779.10 & 103.87 & 0.7500 & 0.0000 & 0.2500 & 0.8930 & 0.0000 & 0.1070 \\
\hline 383 & $\mathrm{CH} 2 \mathrm{Cl} 2$ & & $\mathrm{CCl} 4$ & 805.20 & 107.35 & 0.8000 & 0.0000 & 0.2000 & 0.9160 & 0.0000 & 0.0840 \\
\hline 384 & $\mathrm{CH} 2 \mathrm{Cl} 2$ & & $\mathrm{CCl} 4$ & 831.20 & 110.82 & 0.8500 & 0.0000 & 0.1500 & 0.9380 & 0.0000 & 0.0620 \\
\hline 385 & $\mathrm{CH} 2 \mathrm{Cl} 2$ & & $\mathrm{CCl} 4$ & 858.00 & 114.39 & 0.9000 & 0.0000 & 0.1000 & 0.9590 & 0.0000 & 0.0410 \\
\hline 386 & $\mathrm{CH} 2 \mathrm{Cl} 2$ & & $\mathrm{CCl} 4$ & 868.00 & 115.72 & 0.9200 & 0.0000 & 0.0800 & 0.9670 & 0.0000 & 0.0330 \\
\hline 387 & $\mathrm{CH} 2 \mathrm{Cl} 2$ & & $\mathrm{CCl} 4$ & 884.00 & 117.86 & 0.9500 & 0.0000 & 0.0500 & 0.9790 & 0.0000 & 0.0210 \\
\hline 388 & $\mathrm{CH} 2 \mathrm{Cl} 2$ & & $\mathrm{CCl} 4$ & 894.50 & 119.26 & 0.9700 & 0.0000 & 0.0300 & 0.9870 & 0.0000 & 0.0130 \\
\hline 389 & & $\mathrm{CHCl} 3$ & $\mathrm{CCl} 4$ & 264.80 & 35.30 & 0.0000 & 0.0250 & 0.9750 & 0.0000 & 0.0460 & 0.9540 \\
\hline 390 & & $\mathrm{CHCl} 3$ & $\mathrm{CCl} 4$ & 270.80 & 36.10 & 0.0000 & 0.0500 & 0.9500 & 0.0000 & 0.0880 & 0.9120 \\
\hline 391 & & $\mathrm{CHCl} 3$ & $\mathrm{CCl} 4$ & 282.50 & 37.66 & 0.0000 & 0.1000 & 0.9000 & 0.0000 & 0.1690 & 0.8310 \\
\hline 392 & & $\mathrm{CHCl} 3$ & $\mathrm{CCl} 4$ & 294.00 & 39.20 & 0.0000 & 0.1500 & 0.8500 & 0.0000 & 0.2440 & 0.7560 \\
\hline 393 & & $\mathrm{CHCl} 3$ & $\mathrm{CCl} 4$ & 304.50 & 40.60 & 0.0000 & 0.2000 & 0.8000 & 0.0000 & 0.3120 & 0.6880 \\
\hline 394 & & $\mathrm{CHCl} 3$ & $\mathrm{CCl} 4$ & 314.40 & 41.92 & 0.0000 & 0.2500 & 0.7500 & 0.0000 & 0.3740 & 0.6260 \\
\hline 395 & & $\mathrm{CHCl} 3$ & $\mathrm{CCl} 4$ & 324.30 & 43.24 & 0.0000 & 0.3000 & 0.7000 & 0.0000 & 0.4320 & 0.5680 \\
\hline 396 & & $\mathrm{CHCl} 3$ & $\mathrm{CCl} 4$ & 333.60 & 44.48 & 0.0000 & 0.3500 & 0.6500 & 0.0000 & 0.4900 & 0.5100 \\
\hline 397 & & $\mathrm{CHCl} 3$ & $\mathrm{CCl} 4$ & 342.30 & 45.64 & 0.0000 & 0.4000 & 0.6000 & 0.0000 & 0.5390 & 0.4610 \\
\hline 398 & & $\mathrm{CHCl} 3$ & $\mathrm{CCl} 4$ & 351.30 & 46.84 & 0.0000 & 0.4500 & 0.5500 & 0.0000 & 0.5870 & 0.4130 \\
\hline 399 & & $\mathrm{CHCl} 3$ & $\mathrm{CCl} 4$ & 359.70 & 47.96 & 0.0000 & 0.5000 & 0.5000 & 0.0000 & 0.6300 & 0.3700 \\
\hline 400 & & $\mathrm{CHCl} 3$ & $\mathrm{CCl} 4$ & 368.30 & 49.10 & 0.0000 & 0.5500 & 0.4500 & 0.0000 & 0.6740 & 0.3260 \\
\hline
\end{tabular}




\begin{tabular}{|c|c|c|c|c|c|c|c|c|c|c|c|}
\hline 401 & & $\mathrm{CHCl} 3$ & $\mathrm{CCl} 4$ & 376.60 & 50.21 & 0.0000 & 0.6000 & 0.4000 & 0.0000 & 0.7140 & 0.2860 \\
\hline 402 & & $\mathrm{CHCl} 3$ & $\mathrm{CCl} 4$ & 384.40 & 51.25 & 0.0000 & 0.6500 & 0.3500 & 0.0000 & 0.7550 & 0.2450 \\
\hline 403 & & $\mathrm{CHCl} 3$ & $\mathrm{CCl} 4$ & 392.00 & 52.26 & 0.0000 & 0.7000 & 0.3000 & 0.0000 & 0.7920 & 0.2080 \\
\hline 404 & & $\mathrm{CHCl} 3$ & $\mathrm{CCl} 4$ & 399.00 & 53.20 & 0.0000 & 0.7500 & 0.2500 & 0.0000 & 0.8290 & 0.1710 \\
\hline 405 & & $\mathrm{CHCl} 3$ & $\mathrm{CCl} 4$ & 405.80 & 54.10 & 0.0000 & 0.8000 & 0.2000 & 0.0000 & 0.8640 & 0.1360 \\
\hline 406 & & $\mathrm{CHCl} 3$ & $\mathrm{CCl} 4$ & 412.70 & 55.02 & 0.0000 & 0.8500 & 0.1500 & 0.0000 & 0.8990 & 0.1010 \\
\hline 407 & & $\mathrm{CHCl} 3$ & $\mathrm{CCl} 4$ & 419.70 & 55.96 & 0.0000 & 0.9000 & 0.1000 & 0.0000 & 0.9330 & 0.0670 \\
\hline 408 & & $\mathrm{CHCl} 3$ & $\mathrm{CCl} 4$ & 422.30 & 56.30 & 0.0000 & 0.9200 & 0.0800 & 0.0000 & 0.9470 & 0.0530 \\
\hline 409 & & $\mathrm{CHCl} 3$ & $\mathrm{CCl} 4$ & 426.70 & 56.89 & 0.0000 & 0.9500 & 0.0500 & 0.0000 & 0.9650 & 0.0350 \\
\hline 410 & & $\mathrm{CHCl} 3$ & $\mathrm{CCl} 4$ & 429.30 & 57.24 & 0.0000 & 0.9700 & 0.0300 & 0.0000 & 0.9790 & 0.0210 \\
\hline 411 & C6h12 & Heptane & Toluene & 42.61 & 5.68 & 0.1010 & 0.1001 & 0.7989 & 0.3072 & 0.1483 & 0.5445 \\
\hline 412 & C6h12 & Heptane & Toluene & 46.34 & 6.18 & 0.1009 & 0.2505 & 0.6486 & 0.2628 & 0.3051 & 0.4321 \\
\hline 413 & C6h12 & Heptane & Toluene & 49.12 & 6.55 & 0.1001 & 0.4494 & 0.4505 & 0.2292 & 0.4652 & 0.3056 \\
\hline 414 & C6h12 & Heptane & Toluene & 51.02 & 6.80 & 0.1003 & 0.6492 & 0.2505 & 0.2129 & 0.6075 & 0.1796 \\
\hline 415 & C6h12 & Heptane & Toluene & 50.85 & 6.78 & 0.1007 & 0.7990 & 0.1003 & 0.2091 & 0.7137 & 0.0772 \\
\hline 416 & C6h12 & Heptane & Toluene & 56.00 & 7.47 & 0.2499 & 0.1005 & 0.6496 & 0.5343 & 0.1048 & 0.3609 \\
\hline 417 & C6h12 & Heptane & Toluene & 56.09 & 7.48 & 0.2510 & 0.2493 & 0.4997 & 0.4971 & 0.2273 & 0.2756 \\
\hline 418 & C6h12 & Heptane & Toluene & 59.88 & 7.98 & 0.2509 & 0.4986 & 0.2505 & 0.4492 & 0.3985 & 0.1523 \\
\hline 419 & C6h12 & Heptane & Toluene & 59.32 & 7.91 & 0.2510 & 0.6489 & 0.1001 & 0.4376 & 0.4951 & 0.0673 \\
\hline 420 & C6h12 & Heptane & Toluene & 62.14 & 8.28 & 0.3335 & 0.3325 & 0.3340 & 0.5616 & 0.2575 & 0.1809 \\
\hline 421 & C6h12 & Heptane & Toluene & 69.51 & 9.27 & 0.4495 & 0.0999 & 0.4506 & 0.7052 & 0.0768 & 0.2180 \\
\hline 422 & C6h12 & Heptane & Toluene & 72.13 & 9.62 & 0.4502 & 0.4484 & 0.1014 & 0.6394 & 0.3018 & 0.0588 \\
\hline 423 & C6h12 & Heptane & Toluene & 72.94 & 9.72 & 0.5001 & 0.2500 & 0.2499 & 0.7085 & 0.1680 & 0.1235 \\
\hline 424 & C6h12 & Heptane & Toluene & 83.10 & 11.08 & 0.6502 & 0.0996 & 0.2502 & 0.8172 & 0.0636 & 0.1192 \\
\hline 425 & C6h12 & Heptane & Toluene & 81.86 & 10.91 & 0.6493 & 0.2501 & 0.1006 & 0.7971 & 0.1507 & 0.0522 \\
\hline 426 & C6h12 & Heptane & Toluene & 88.99 & 11.86 & 0.7992 & 0.0997 & 0.1011 & 0.8905 & 0.0589 & 0.0506 \\
\hline 427 & C6h12 & Heptane & & 47.98 & 6.40 & 0.0468 & 0.9532 & 0.0000 & 0.1040 & 0.8960 & 0.0000 \\
\hline
\end{tabular}




\begin{tabular}{|c|c|c|c|c|c|c|c|c|c|c|c|}
\hline 428 & C6h12 & Heptane & & 51.55 & 6.87 & 0.1010 & 0.8990 & 0.0000 & 0.2074 & 0.7926 & 0.0000 \\
\hline 429 & C6h12 & Heptane & & 57.17 & 7.62 & 0.1877 & 0.8123 & 0.0000 & 0.3469 & 0.6531 & 0.0000 \\
\hline 430 & C6h12 & Heptane & & 63.50 & 8.47 & 0.3005 & 0.6995 & 0.0000 & 0.4891 & 0.5109 & 0.0000 \\
\hline 431 & C6h12 & Heptane & & 67.52 & 9.00 & 0.3821 & 0.6179 & 0.0000 & 0.5764 & 0.4236 & 0.0000 \\
\hline 432 & C6h12 & Heptane & & 74.45 & 9.93 & 0.5001 & 0.4999 & 0.0000 & 0.6799 & 0.3201 & 0.0000 \\
\hline 433 & C6h12 & Heptane & & 77.43 & 10.32 & 0.5819 & 0.4181 & 0.0000 & 0.7469 & 0.2531 & 0.0000 \\
\hline 434 & C6h12 & Heptane & & 83.26 & 11.10 & 0.6999 & 0.3001 & 0.0000 & 0.8252 & 0.1748 & 0.0000 \\
\hline 435 & C6h12 & Heptane & & 87.10 & 11.61 & 0.7879 & 0.2121 & 0.0000 & 0.8821 & 0.1179 & 0.0000 \\
\hline 436 & C6h12 & Heptane & & 92.45 & 12.33 & 0.8998 & 0.1002 & 0.0000 & 0.9439 & 0.0561 & 0.0000 \\
\hline 437 & C6h12 & Heptane & & 95.03 & 12.67 & 0.9459 & 0.0541 & 0.0000 & 0.9706 & 0.0294 & 0.0000 \\
\hline 438 & C6h12 & & Toluene & 34.36 & 4.58 & 0.0505 & 0.0000 & 0.9495 & 0.2157 & 0.0000 & 0.7843 \\
\hline 439 & C6h12 & & Toluene & 38.89 & 5.18 & 0.1013 & 0.0000 & 0.8987 & 0.3610 & 0.0000 & 0.6390 \\
\hline 440 & C6h12 & & Toluene & 49.03 & 6.54 & 0.1989 & 0.0000 & 0.8011 & 0.5313 & 0.0000 & 0.4687 \\
\hline 441 & C6h12 & & Toluene & 58.24 & 7.76 & 0.2836 & 0.0000 & 0.7164 & 0.6085 & 0.0000 & 0.3915 \\
\hline 442 & C6h12 & & Toluene & 65.25 & 8.70 & 0.4002 & 0.0000 & 0.5998 & 0.7139 & 0.0000 & 0.2861 \\
\hline 443 & C6h12 & & Toluene & 70.73 & 9.43 & 0.5000 & 0.0000 & 0.5000 & 0.7735 & 0.0000 & 0.2265 \\
\hline 444 & C6h12 & & Toluene & 77.61 & 10.35 & 0.6004 & 0.0000 & 0.3996 & 0.8224 & 0.0000 & 0.1776 \\
\hline 445 & C6h12 & & Toluene & 82.12 & 10.95 & 0.7000 & 0.0000 & 0.3000 & 0.8665 & 0.0000 & 0.1335 \\
\hline 446 & C6h12 & & Toluene & 86.71 & 11.56 & 0.7998 & 0.0000 & 0.2002 & 0.9123 & 0.0000 & 0.0877 \\
\hline 447 & C6h12 & & Toluene & 91.64 & 12.22 & 0.8996 & 0.0000 & 0.1004 & 0.9531 & 0.0000 & 0.0469 \\
\hline 448 & C6h12 & & Toluene & 94.83 & 12.64 & 0.9501 & 0.0000 & 0.0499 & 0.9763 & 0.0000 & 0.0237 \\
\hline 449 & & Heptane & Toluene & 29.69 & 3.96 & 0.0000 & 0.0500 & 0.9500 & 0.0000 & 0.1209 & 0.8791 \\
\hline 450 & & Heptane & Toluene & 31.80 & 4.24 & 0.0000 & 0.0999 & 0.9001 & 0.0000 & 0.2123 & 0.7877 \\
\hline 451 & & Heptane & Toluene & 35.58 & 4.74 & 0.0000 & 0.1991 & 0.8009 & 0.0000 & 0.3465 & 0.6535 \\
\hline 452 & & Heptane & Toluene & 38.67 & 5.16 & 0.0000 & 0.3003 & 0.6997 & 0.0000 & 0.4435 & 0.5565 \\
\hline 453 & & Heptane & Toluene & 39.95 & 5.33 & 0.0000 & 0.3979 & 0.6021 & 0.0000 & 0.5304 & 0.4696 \\
\hline 454 & & Heptane & Toluene & 40.23 & 5.36 & 0.0000 & 0.4999 & 0.5001 & 0.0000 & 0.6147 & 0.3853 \\
\hline
\end{tabular}




\begin{tabular}{|c|c|c|c|c|c|c|c|c|c|c|c|}
\hline 455 & & Heptane & Toluene & 42.07 & 5.61 & 0.0000 & 0.6002 & 0.3998 & 0.0000 & 0.6855 & 0.3145 \\
\hline 456 & & Heptane & Toluene & 46.09 & 6.14 & 0.0000 & 0.6989 & 0.3011 & 0.0000 & 0.7574 & 0.2426 \\
\hline 457 & & Heptane & Toluene & 43.82 & 5.84 & 0.0000 & 0.7985 & 0.2015 & 0.0000 & 0.8320 & 0.1680 \\
\hline 458 & & Heptane & Toluene & 45.31 & 6.04 & 0.0000 & 0.8984 & 0.1016 & 0.0000 & 0.9111 & 0.0889 \\
\hline 459 & & Heptane & Toluene & 45.30 & 6.04 & 0.0000 & 0.9494 & 0.0506 & 0.0000 & 0.9518 & 0.0482 \\
\hline 460 & Methanol & $\mathrm{CCl} 4$ & Benzene & 665.26 & 88.69 & 0.1880 & 0.1960 & 0.6160 & 0.5152 & 0.1387 & 0.3461 \\
\hline 461 & Methanol & $\mathrm{CCl} 4$ & Benzene & 690.29 & 92.03 & 0.1983 & 0.3961 & 0.4056 & 0.5130 & 0.2624 & 0.2246 \\
\hline 462 & Methanol & $\mathrm{CCl} 4$ & Benzene & 689.67 & 91.95 & 0.1982 & 0.3963 & 0.4055 & 0.5109 & 0.2628 & 0.2263 \\
\hline 463 & Methanol & $\mathrm{CCl} 4$ & Benzene & 706.51 & 94.19 & 0.1945 & 0.5922 & 0.2133 & 0.5086 & 0.3733 & 0.1181 \\
\hline 464 & Methanol & $\mathrm{CCl} 4$ & Benzene & 711.17 & 94.81 & 0.3590 & 0.3230 & 0.3180 & 0.5397 & 0.2539 & 0.2064 \\
\hline 465 & Methanol & $\mathrm{CCl} 4$ & Benzene & 717.20 & 95.62 & 0.5557 & 0.2134 & 0.2309 & 0.5672 & 0.2316 & 0.2012 \\
\hline 466 & Methanol & $\mathrm{CCl} 4$ & Benzene & 703.34 & 93.77 & 0.7515 & 0.1115 & 0.1370 & 0.6209 & 0.1925 & 0.1866 \\
\hline 467 & Methanol & $\mathrm{CCl} 4$ & Benzene & 680.86 & 90.77 & 0.8433 & 0.0814 & 0.0753 & 0.6736 & 0.1890 & 0.1374 \\
\hline 468 & Methanol & $\mathrm{CCl} 4$ & & 580.66 & 77.41 & 0.0254 & 0.9746 & 0.0000 & 0.3619 & 0.6381 & 0.0000 \\
\hline 469 & Methanol & $\mathrm{CCl} 4$ & & 591.16 & 78.81 & 0.0579 & 0.9421 & 0.0000 & 0.3639 & 0.6361 & 0.0000 \\
\hline 470 & Methanol & $\mathrm{CCl} 4$ & & 716.95 & 95.59 & 0.1493 & 0.8507 & 0.0000 & 0.4981 & 0.5019 & 0.0000 \\
\hline 471 & Methanol & $\mathrm{CCl} 4$ & & 741.36 & 98.84 & 0.3647 & 0.6353 & 0.0000 & 0.5284 & 0.4716 & 0.0000 \\
\hline 472 & Methanol & $\mathrm{CCl} 4$ & & 745.60 & 99.41 & 0.4893 & 0.5107 & 0.0000 & 0.5431 & 0.4569 & 0.0000 \\
\hline 473 & Methanol & $\mathrm{CCl} 4$ & & 745.72 & 99.42 & 0.4956 & 0.5044 & 0.0000 & 0.5438 & 0.4562 & 0.0000 \\
\hline 474 & Methanol & $\mathrm{CCl} 4$ & & 744.54 & 99.26 & 0.6448 & 0.3552 & 0.0000 & 0.5686 & 0.4314 & 0.0000 \\
\hline 475 & Methanol & $\mathrm{CCl} 4$ & & 724.28 & 96.56 & 0.7903 & 0.2097 & 0.0000 & 0.6187 & 0.3813 & 0.0000 \\
\hline 476 & Methanol & $\mathrm{CCl} 4$ & & 658.37 & 87.78 & 0.9087 & 0.0913 & 0.0000 & 0.7337 & 0.2663 & 0.0000 \\
\hline 477 & Methanol & & Benzene & 465.84 & 62.11 & 0.0304 & 0.0000 & 0.9696 & 0.3019 & 0.0000 & 0.6981 \\
\hline 478 & Methanol & & Benzene & 527.12 & 70.28 & 0.0493 & 0.0000 & 0.9507 & 0.4051 & 0.0000 & 0.5949 \\
\hline 479 & Methanol & & Benzene & 570.00 & 75.99 & 0.1000 & 0.0000 & 0.9000 & 0.4560 & 0.0000 & 0.5440 \\
\hline 480 & Methanol & & Benzene & 597.48 & 79.66 & 0.1031 & 0.0000 & 0.8969 & 0.4841 & 0.0000 & 0.5159 \\
\hline 481 & Methanol & & Benzene & 637.00 & 84.93 & 0.2000 & 0.0000 & 0.8000 & 0.5320 & 0.0000 & 0.4680 \\
\hline
\end{tabular}




\begin{tabular}{|c|c|c|c|c|c|c|c|c|c|c|c|}
\hline 482 & Methanol & & Benzene & 664.24 & 88.56 & 0.3297 & 0.0000 & 0.6703 & 0.5540 & 0.0000 & 0.4460 \\
\hline 483 & Methanol & & Benzene & 670.00 & 89.33 & 0.4000 & 0.0000 & 0.6000 & 0.5750 & 0.0000 & 0.4250 \\
\hline 484 & Methanol & & Benzene & 675.62 & 90.08 & 0.4874 & 0.0000 & 0.5126 & 0.5845 & 0.0000 & 0.4155 \\
\hline 485 & Methanol & & Benzene & 675.99 & 90.12 & 0.4984 & 0.0000 & 0.5016 & 0.5858 & 0.0000 & 0.4142 \\
\hline 486 & Methanol & & Benzene & 675.00 & 89.99 & 0.6000 & 0.0000 & 0.4000 & 0.6080 & 0.0000 & 0.3920 \\
\hline 487 & Methanol & & Benzene & 678.44 & 90.45 & 0.6079 & 0.0000 & 0.3921 & 0.6078 & 0.0000 & 0.3922 \\
\hline 488 & Methanol & & Benzene & 664.91 & 88.65 & 0.7896 & 0.0000 & 0.2104 & 0.6716 & 0.0000 & 0.3284 \\
\hline 489 & Methanol & & Benzene & 664.00 & 88.53 & 0.8000 & 0.0000 & 0.2000 & 0.6710 & 0.0000 & 0.3290 \\
\hline 490 & Methanol & & Benzene & 622.29 & 82.97 & 0.9014 & 0.0000 & 0.0986 & 0.7697 & 0.0000 & 0.2303 \\
\hline 491 & & $\mathrm{CCl} 4$ & Benzene & 335.85 & 44.78 & 0.0000 & 0.1000 & 0.9000 & 0.0000 & 0.1222 & 0.8778 \\
\hline 492 & & $\mathrm{CCl} 4$ & Benzene & 347.43 & 46.32 & 0.0000 & 0.2500 & 0.7500 & 0.0000 & 0.2888 & 0.7112 \\
\hline 493 & & $\mathrm{CCl} 4$ & Benzene & 350.84 & 46.77 & 0.0000 & 0.3000 & 0.7000 & 0.0000 & 0.3405 & 0.6595 \\
\hline 494 & & $\mathrm{CCl} 4$ & Benzene & 356.94 & 47.59 & 0.0000 & 0.4000 & 0.6000 & 0.0000 & 0.4393 & 0.5607 \\
\hline 495 & & $\mathrm{CCl} 4$ & Benzene & 362.02 & 48.27 & 0.0000 & 0.5000 & 0.5000 & 0.0000 & 0.5333 & 0.4667 \\
\hline 496 & & $\mathrm{CCl} 4$ & Benzene & 366.03 & 48.80 & 0.0000 & 0.6000 & 0.4000 & 0.0000 & 0.6240 & 0.3760 \\
\hline 497 & & $\mathrm{CCl} 4$ & Benzene & 369.81 & 49.30 & 0.0000 & 0.7500 & 0.2500 & 0.0000 & 0.7583 & 0.2417 \\
\hline 498 & & $\mathrm{CCl} 4$ & Benzene & 370.41 & 49.38 & 0.0000 & 0.9000 & 0.1000 & 0.0000 & 0.8979 & 0.1021 \\
\hline 499 & Methyl acetate & Chloroform & Benzene & 326.00 & 43.46 & 0.1030 & 0.0760 & 0.8210 & 0.2190 & 0.0910 & 0.6900 \\
\hline 500 & Methyl acetate & Chloroform & Benzene & 332.70 & 44.36 & 0.1200 & 0.0730 & 0.8070 & 0.2520 & 0.0820 & 0.6660 \\
\hline 501 & Methyl acetate & Chloroform & Benzene & 348.40 & 46.45 & 0.1630 & 0.0680 & 0.7690 & 0.3130 & 0.0740 & 0.6130 \\
\hline 502 & Methyl acetate & Chloroform & Benzene & 385.90 & 51.45 & 0.2570 & 0.0730 & 0.6700 & 0.4380 & 0.0670 & 0.4950 \\
\hline 503 & Methyl acetate & Chloroform & Benzene & 486.30 & 64.83 & 0.5880 & 0.0500 & 0.3620 & 0.7400 & 0.0310 & 0.2290 \\
\hline 504 & Methyl acetate & Chloroform & Benzene & 553.80 & 73.83 & 0.8200 & 0.0180 & 0.1620 & 0.8920 & 0.0100 & 0.0980 \\
\hline 505 & Methyl acetate & Chloroform & Benzene & 315.20 & 42.02 & 0.0540 & 0.1640 & 0.7820 & 0.1050 & 0.2200 & 0.6750 \\
\hline 506 & Methyl acetate & Chloroform & Benzene & 324.30 & 43.24 & 0.0770 & 0.1690 & 0.7540 & 0.1490 & 0.2150 & 0.6360 \\
\hline 507 & Methyl acetate & Chloroform & Benzene & 341.40 & 45.52 & 0.1250 & 0.1620 & 0.7130 & 0.2310 & 0.1920 & 0.5770 \\
\hline 508 & Methyl acetate & Chloroform & Benzene & 368.40 & 49.12 & 0.2000 & 0.1530 & 0.6470 & 0.3390 & 0.1600 & 0.5010 \\
\hline
\end{tabular}




\begin{tabular}{|c|c|c|c|c|c|c|c|c|c|c|c|}
\hline 509 & Methyl acetate & Chloroform & Benzene & 401.20 & 53.49 & 0.2990 & 0.1390 & 0.5620 & 0.4710 & 0.1250 & 0.4040 \\
\hline 510 & Methyl acetate & Chloroform & Benzene & 447.00 & 59.60 & 0.4220 & 0.1190 & 0.4590 & 0.6050 & 0.0880 & 0.3070 \\
\hline 511 & Methyl acetate & Chloroform & Benzene & 510.30 & 68.03 & 0.6670 & 0.0730 & 0.2600 & 0.7930 & 0.0390 & 0.1680 \\
\hline 512 & Methyl acetate & Chloroform & Benzene & 330.80 & 44.10 & 0.0620 & 0.2440 & 0.6940 & 0.1080 & 0.3180 & 0.5740 \\
\hline 513 & Methyl acetate & Chloroform & Benzene & 341.30 & 45.50 & 0.0930 & 0.2400 & 0.6670 & 0.1620 & 0.3010 & 0.5370 \\
\hline 514 & Methyl acetate & Chloroform & Benzene & 378.80 & 50.50 & 0.2110 & 0.2190 & 0.5700 & 0.3380 & 0.2290 & 0.4330 \\
\hline 515 & Methyl acetate & Chloroform & Benzene & 412.90 & 55.05 & 0.3380 & 0.1980 & 0.4640 & 0.4950 & 0.1740 & 0.3310 \\
\hline 516 & Methyl acetate & Chloroform & Benzene & 513.80 & 68.50 & 0.6870 & 0.1020 & 0.2110 & 0.8000 & 0.0620 & 0.1380 \\
\hline 517 & Methyl acetate & Chloroform & Benzene & 562.60 & 75.01 & 0.8830 & 0.0380 & 0.0790 & 0.9310 & 0.0200 & 0.0490 \\
\hline 518 & Methyl acetate & Chloroform & Benzene & 563.80 & 75.17 & 0.8670 & 0.0440 & 0.0890 & 0.9190 & 0.0240 & 0.0570 \\
\hline 519 & Methyl acetate & Chloroform & Benzene & 348.40 & 46.45 & 0.0660 & 0.3380 & 0.5960 & 0.1020 & 0.4330 & 0.4650 \\
\hline 520 & Methyl acetate & Chloroform & Benzene & 354.60 & 47.28 & 0.1060 & 0.3140 & 0.5800 & 0.1730 & 0.3910 & 0.4360 \\
\hline 3 & Methyl acetate & Chloroform & Benzene & 369.50 & 49.26 & 0.1400 & 0.3030 & 0.5570 & 0.2270 & 0.3500 & 0.4230 \\
\hline & Methyl acetate & Chloroform & Benzene & 388.60 & 51.81 & 0.2340 & 0.2940 & 0.4720 & 0.3450 & 0.3020 & 0.3530 \\
\hline & Methyl acetate & Chloroform & Benzene & 415.90 & 55.45 & 0.3460 & 0.2590 & 0.3950 & 0.4850 & 0.2300 & 0.2850 \\
\hline & Methyl acetate & Chloroform & Benzene & 459.50 & 61.26 & 0.4980 & 0.2120 & 0.2900 & 0.6410 & 0.1600 & 0.1990 \\
\hline & Methyl acetate & Chloroform & Benzene & 513.60 & 68.47 & 0.7010 & 0.1270 & 0.1720 & 0.8080 & 0.0800 & 0.1120 \\
\hline & Methyl acetate & Chloroform & Benzene & 556.60 & 74.21 & 0.8560 & 0.0620 & 0.0820 & 0.9150 & 0.0340 & 0.0510 \\
\hline 527 & Methyl acetate & Chloroform & Benzene & 364.80 & 48.64 & 0.0710 & 0.4200 & 0.5090 & 0.0960 & 0.5270 & 0.3770 \\
\hline 528 & Methyl acetate & Chloroform & Benzene & 368.90 & 49.18 & 0.1070 & 0.4030 & 0.4900 & 0.1510 & 0.4870 & 0.3620 \\
\hline 325 & Methyl acetate & Chloroform & Benzene & 382.70 & 51.02 & 0.1580 & 0.3740 & 0.4680 & 0.2210 & 0.4310 & 0.3480 \\
\hline טנד & Methyl acetate & Chloroform & Benzene & 407.90 & 54.38 & 0.2910 & 0.3440 & 0.3650 & 0.3940 & 0.3380 & 0.2680 \\
\hline 531 & Methyl acetate & Chloroform & Benzene & 422.60 & 56.34 & 0.3680 & 0.3150 & 0.3170 & 0.4750 & 0.2900 & 0.2350 \\
\hline & Methyl acetate & Chloroform & Benzene & 456.90 & 60.91 & 0.4860 & 0.2580 & 0.2560 & 0.6210 & 0.2020 & 0.1770 \\
\hline & Methyl acetate & Chloroform & Benzene & 514.60 & 68.61 & 0.6790 & 0.1700 & 0.1510 & 0.7910 & 0.1100 & 0.0990 \\
\hline & Methyl acetate & Chloroform & Benzene & 565.80 & 75.43 & 0.8860 & 0.0620 & 0.0520 & 0.9340 & 0.0330 & 0.0330 \\
\hline & Methyl acetate & Chloroform & Benzene & 384.70 & 51.29 & 0.0760 & 0.5060 & 0.4180 & 0.0960 & 0.6190 & 0.2850 \\
\hline
\end{tabular}




\begin{tabular}{|c|c|c|c|c|c|c|c|c|c|c|c|}
\hline 536 & Methyl acetate & Chloroform & Benzene & 386.40 & 51.52 & 0.1200 & 0.4790 & 0.4010 & 0.1280 & 0.5710 & 0.3010 \\
\hline 537 & Methyl acetate & Chloroform & Benzene & 392.00 & 52.26 & 0.1730 & 0.4650 & 0.3620 & 0.2150 & 0.5270 & 0.2580 \\
\hline 538 & Methyl acetate & Chloroform & Benzene & 410.10 & 54.68 & 0.2640 & 0.4270 & 0.3090 & 0.3380 & 0.4360 & 0.2260 \\
\hline 539 & Methyl acetate & Chloroform & Benzene & 430.30 & 57.37 & 0.3670 & 0.3760 & 0.2570 & 0.4670 & 0.3480 & 0.1850 \\
\hline 540 & Methyl acetate & Chloroform & Benzene & 463.10 & 61.74 & 0.5060 & 0.3000 & 0.1940 & 0.6280 & 0.2340 & 0.1380 \\
\hline 541 & Methyl acetate & Chloroform & Benzene & 563.70 & 75.15 & 0.8860 & 0.0740 & 0.0400 & 0.9350 & 0.0380 & 0.0270 \\
\hline 542 & Methyl acetate & Chloroform & Benzene & 408.00 & 54.40 & 0.0780 & 0.6010 & 0.3210 & 0.0830 & 0.7160 & 0.2010 \\
\hline 543 & Methyl acetate & Chloroform & Benzene & 405.70 & 54.09 & 0.1840 & 0.5450 & 0.2710 & 0.2040 & 0.6080 & 0.1880 \\
\hline 544 & Methyl acetate & Chloroform & Benzene & 420.10 & 56.01 & 0.2700 & 0.4960 & 0.2340 & 0.3180 & 0.5180 & 0.1640 \\
\hline 545 & Methyl acetate & Chloroform & Benzene & 434.70 & 57.96 & 0.3760 & 0.4350 & 0.1890 & 0.4530 & 0.4100 & 0.1370 \\
\hline 546 & Methyl acetate & Chloroform & Benzene & 459.60 & 61.27 & 0.5180 & 0.3430 & 0.1390 & 0.6250 & 0.2770 & 0.0980 \\
\hline 547 & Methyl acetate & Chloroform & Benzene & 509.70 & 67.95 & 0.6700 & 0.2390 & 0.0910 & 0.7750 & 0.1630 & 0.0620 \\
\hline 548 & Methyl acetate & Chloroform & Benzene & 561.70 & 74.89 & 0.8900 & 0.0820 & 0.0280 & 0.9380 & 0.0460 & 0.0160 \\
\hline 549 & Methyl acetate & Chloroform & Benzene & 428.90 & 57.18 & 0.0920 & 0.6670 & 0.2410 & 0.0870 & 0.7680 & 0.1450 \\
\hline 550 & Methyl acetate & Chloroform & Benzene & 427.10 & 56.94 & 0.1400 & 0.6440 & 0.2160 & 0.1360 & 0.7290 & 0.1350 \\
\hline 551 & Methyl acetate & Chloroform & Benzene & 424.20 & 56.56 & 0.1910 & 0.6280 & 0.1810 & 0.1890 & 0.6940 & 0.1170 \\
\hline 552 & Methyl acetate & Chloroform & Benzene & 424.80 & 56.64 & 0.2790 & 0.5380 & 0.1830 & 0.3120 & 0.5600 & 0.1280 \\
\hline 553 & Methyl acetate & Chloroform & Benzene & 436.90 & 58.25 & 0.3830 & 0.4900 & 0.1270 & 0.4420 & 0.4680 & 0.0900 \\
\hline 554 & Methyl acetate & Chloroform & Benzene & 462.00 & 61.59 & 0.5170 & 0.4030 & 0.0800 & 0.6050 & 0.3380 & 0.0570 \\
\hline 555 & Methyl acetate & Chloroform & Benzene & 506.60 & 67.54 & 0.7010 & 0.2390 & 0.0600 & 0.8000 & 0.1600 & 0.0400 \\
\hline 556 & Methyl acetate & Chloroform & Benzene & 553.20 & 73.75 & 0.8650 & 0.1130 & 0.0220 & 0.9220 & 0.0640 & 0.0140 \\
\hline 557 & Methyl acetate & Chloroform & Benzene & 463.40 & 61.78 & 0.0900 & 0.7980 & 0.1120 & 0.0690 & 0.8740 & 0.0570 \\
\hline 558 & Methyl acetate & Chloroform & Benzene & 453.00 & 60.40 & 0.1450 & 0.7480 & 0.1070 & 0.1200 & 0.8190 & 0.0610 \\
\hline 559 & Methyl acetate & Chloroform & Benzene & 444.20 & 59.22 & 0.2080 & 0.6900 & 0.1020 & 0.1900 & 0.7460 & 0.0640 \\
\hline 560 & Methyl acetate & Chloroform & Benzene & 442.20 & 58.96 & 0.2880 & 0.6310 & 0.0810 & 0.2940 & 0.6570 & 0.0490 \\
\hline 561 & Methyl acetate & Chloroform & Benzene & 448.40 & 59.78 & 0.3960 & 0.5440 & 0.0600 & 0.4310 & 0.5260 & 0.0430 \\
\hline 562 & Methyl acetate & Chloroform & Benzene & 466.50 & 62.19 & 0.5220 & 0.4320 & 0.0460 & 0.6020 & 0.3670 & 0.0310 \\
\hline
\end{tabular}




\begin{tabular}{|c|c|c|c|c|c|c|c|c|c|c|c|}
\hline 563 & Methyl acetate & Chloroform & Benzene & 508.40 & 67.78 & 0.6750 & 0.2970 & 0.0280 & 0.7730 & 0.2100 & 0.0170 \\
\hline 564 & Methyl acetate & Chloroform & Benzene & 356.60 & 47.54 & 0.1530 & 0.2360 & 0.6110 & 0.2540 & 0.2690 & 0.4770 \\
\hline 565 & Methyl acetate & Chloroform & & 500.30 & 66.70 & 0.0640 & 0.9360 & 0.0000 & 0.0400 & 0.9600 & 0.0000 \\
\hline 566 & Methyl acetate & Chloroform & & 482.00 & 64.26 & 0.1340 & 0.8660 & 0.0000 & 0.0890 & 0.9110 & 0.0000 \\
\hline 567 & Methyl acetate & Chloroform & & 472.10 & 62.94 & 0.1780 & 0.8220 & 0.0000 & 0.1310 & 0.8690 & 0.0000 \\
\hline 568 & Methyl acetate & Chloroform & & 455.60 & 60.74 & 0.2580 & 0.7420 & 0.0000 & 0.2200 & 0.7800 & 0.0000 \\
\hline 569 & Methyl acetate & Chloroform & & 450.10 & 60.01 & 0.3140 & 0.6860 & 0.0000 & 0.3080 & 0.6920 & 0.0000 \\
\hline 570 & Methyl acetate & Chloroform & & 449.10 & 59.88 & 0.3560 & 0.6440 & 0.0000 & 0.3540 & 0.6460 & 0.0000 \\
\hline 571 & Methyl acetate & Chloroform & & 450.60 & 60.08 & 0.3940 & 0.6060 & 0.0000 & 0.4050 & 0.5950 & 0.0000 \\
\hline 572 & Methyl acetate & Chloroform & & 453.80 & 60.50 & 0.4380 & 0.5620 & 0.0000 & 0.4700 & 0.5300 & 0.0000 \\
\hline 573 & Methyl acetate & Chloroform & & 458.10 & 61.07 & 0.4770 & 0.5230 & 0.0000 & 0.5260 & 0.4740 & 0.0000 \\
\hline 574 & Methyl acetate & Chloroform & & 465.30 & 62.03 & 0.5250 & 0.4750 & 0.0000 & 0.5840 & 0.4160 & 0.0000 \\
\hline 575 & Methyl acetate & Chloroform & & 483.60 & 64.47 & 0.6120 & 0.3880 & 0.0000 & 0.6980 & 0.3020 & 0.0000 \\
\hline 576 & Methyl acetate & Chloroform & & 508.00 & 67.73 & 0.7070 & 0.2930 & 0.0000 & 0.7960 & 0.2040 & 0.0000 \\
\hline 577 & Methyl acetate & Chloroform & & 512.80 & 68.37 & 0.7260 & 0.2740 & 0.0000 & 0.8140 & 0.1860 & 0.0000 \\
\hline 578 & Methyl acetate & Chloroform & & 518.00 & 69.06 & 0.7470 & 0.2530 & 0.0000 & 0.8350 & 0.1650 & 0.0000 \\
\hline 579 & Methyl acetate & Chloroform & & 535.20 & 71.35 & 0.8150 & 0.1850 & 0.0000 & 0.8860 & 0.1140 & 0.0000 \\
\hline 580 & Methyl acetate & Chloroform & & 571.90 & 76.25 & 0.9350 & 0.0650 & 0.0000 & 0.9660 & 0.0340 & 0.0000 \\
\hline 581 & Methyl acetate & & Benzene & 309.80 & 41.30 & 0.0800 & 0.0000 & 0.9200 & 0.1940 & 0.0000 & 0.8060 \\
\hline 582 & Methyl acetate & & Benzene & 364.40 & 48.58 & 0.1910 & 0.0000 & 0.8090 & 0.3810 & 0.0000 & 0.6190 \\
\hline 583 & Methyl acetate & & Benzene & 387.00 & 51.60 & 0.2540 & 0.0000 & 0.7460 & 0.4610 & 0.0000 & 0.5390 \\
\hline 584 & Methyl acetate & & Benzene & 432.70 & 57.69 & 0.3710 & 0.0000 & 0.6290 & 0.5770 & 0.0000 & 0.4230 \\
\hline 585 & Methyl acetate & & Benzene & 445.70 & 59.42 & 0.4140 & 0.0000 & 0.5860 & 0.6140 & 0.0000 & 0.3860 \\
\hline 586 & Methyl acetate & & Benzene & 464.40 & 61.91 & 0.4660 & 0.0000 & 0.5340 & 0.6570 & 0.0000 & 0.3430 \\
\hline 587 & Methyl acetate & & Benzene & 483.70 & 64.49 & 0.5480 & 0.0000 & 0.4520 & 0.7160 & 0.0000 & 0.2840 \\
\hline 588 & Methyl acetate & & Benzene & 513.20 & 68.42 & 0.6500 & 0.0000 & 0.3500 & 0.7840 & 0.0000 & 0.2160 \\
\hline 500 & Methyl acetate & & Benzene & 525.10 & 70.01 & 0.7020 & 0.0000 & 0.2980 & 0.8160 & 0.0000 & 0.1840 \\
\hline
\end{tabular}




\begin{tabular}{|c|c|c|c|c|c|c|c|c|c|c|c|}
\hline 590 & Methyl acetate & & Benzene & 540.00 & 71.99 & 0.7590 & 0.0000 & 0.2410 & 0.8510 & 0.0000 & 0.1490 \\
\hline 591 & Methyl acetate & & Benzene & 555.30 & 74.03 & 0.8110 & 0.0000 & 0.1890 & 0.8840 & 0.0000 & 0.1160 \\
\hline 592 & Methyl acetate & & Benzene & 581.20 & 77.49 & 0.9270 & 0.0000 & 0.0730 & 0.9550 & 0.0000 & 0.0450 \\
\hline 593 & & Chloroform & Benzene & 276.20 & 36.82 & 0.0000 & 0.0340 & 0.9660 & 0.0000 & 0.0550 & 0.9450 \\
\hline 594 & & Chloroform & Benzene & 278.80 & 37.17 & 0.0000 & 0.0550 & 0.9450 & 0.0000 & 0.0770 & 0.9230 \\
\hline 595 & & Chloroform & Benzene & 288.60 & 38.48 & 0.0000 & 0.1210 & 0.8790 & 0.0000 & 0.1820 & 0.8180 \\
\hline 596 & & Chloroform & Benzene & 294.70 & 39.29 & 0.0000 & 0.1490 & 0.8510 & 0.0000 & 0.2180 & 0.7820 \\
\hline 597 & & Chloroform & Benzene & 302.30 & 40.30 & 0.0000 & 0.1840 & 0.8160 & 0.0000 & 0.2710 & 0.7290 \\
\hline 598 & & Chloroform & Benzene & 317.60 & 42.34 & 0.0000 & 0.2760 & 0.7240 & 0.0000 & 0.3950 & 0.6050 \\
\hline 599 & & Chloroform & Benzene & 329.40 & 43.92 & 0.0000 & 0.3140 & 0.6860 & 0.0000 & 0.4380 & 0.5620 \\
\hline 600 & & Chloroform & Benzene & 348.40 & 46.45 & 0.0000 & 0.4060 & 0.5940 & 0.0000 & 0.5540 & 0.4460 \\
\hline 601 & & Chloroform & Benzene & 353.00 & 47.06 & 0.0000 & 0.4340 & 0.5660 & 0.0000 & 0.5810 & 0.4190 \\
\hline 602 & & Chloroform & Benzene & 363.60 & 48.48 & 0.0000 & 0.4790 & 0.5210 & 0.0000 & 0.6360 & 0.3640 \\
\hline 603 & & Chloroform & Benzene & 384.00 & 51.20 & 0.0000 & 0.5470 & 0.4530 & 0.0000 & 0.7020 & 0.2980 \\
\hline 604 & & Chloroform & Benzene & 388.40 & 51.78 & 0.0000 & 0.5640 & 0.4360 & 0.0000 & 0.7150 & 0.2850 \\
\hline 605 & & Chloroform & Benzene & 410.80 & 54.77 & 0.0000 & 0.6470 & 0.3530 & 0.0000 & 0.7910 & 0.2090 \\
\hline 606 & & Chloroform & Benzene & 424.80 & 56.64 & 0.0000 & 0.6930 & 0.3070 & 0.0000 & 0.8240 & 0.1760 \\
\hline 607 & & Chloroform & Benzene & 437.60 & 58.34 & 0.0000 & 0.7460 & 0.2540 & 0.0000 & 0.8630 & 0.1370 \\
\hline 608 & & Chloroform & Benzene & 467.70 & 62.35 & 0.0000 & 0.8330 & 0.1670 & 0.0000 & 0.9220 & 0.0780 \\
\hline 609 & & Chloroform & Benzene & 478.20 & 63.75 & 0.0000 & 0.8680 & 0.1320 & 0.0000 & 0.9420 & 0.0580 \\
\hline 610 & & Chloroform & Benzene & 499.80 & 66.63 & 0.0000 & 0.9460 & 0.0540 & 0.0000 & 0.9800 & 0.0200 \\
\hline 611 & Water & $\mathrm{CH} 3 \mathrm{OH}$ & $\mathrm{C} 2 \mathrm{H} 5 \mathrm{OH}$ & 64.59 & 8.61 & 0.0955 & 0.0992 & 0.8053 & 0.0770 & 0.1830 & 0.7400 \\
\hline 612 & Water & $\mathrm{CH} 3 \mathrm{OH}$ & $\mathrm{C} 2 \mathrm{H} 5 \mathrm{OH}$ & 70.87 & 9.45 & 0.1030 & 0.1974 & 0.6996 & 0.0720 & 0.3350 & 0.5930 \\
\hline 613 & Water & $\mathrm{CH} 3 \mathrm{OH}$ & $\mathrm{C} 2 \mathrm{H} 5 \mathrm{OH}$ & 103.98 & 13.86 & 0.0940 & 0.7047 & 0.2013 & 0.0350 & 0.8470 & 0.1180 \\
\hline 614 & Water & $\mathrm{CH} 3 \mathrm{OH}$ & $\mathrm{C} 2 \mathrm{H} 5 \mathrm{OH}$ & 110.71 & 14.76 & 0.0966 & 0.8067 & 0.0967 & 0.0320 & 0.9150 & 0.0530 \\
\hline 615 & Water & $\mathrm{CH} 3 \mathrm{OH}$ & $\mathrm{C} 2 \mathrm{H} 5 \mathrm{OH}$ & 72.18 & 9.62 & 0.1513 & 0.2243 & 0.6244 & 0.1000 & 0.3700 & 0.5300 \\
\hline 616 & Water & $\mathrm{CH} 3 \mathrm{OH}$ & $\mathrm{C} 2 \mathrm{H} 5 \mathrm{OH}$ & 76.18 & 10.16 & 0.1445 & 0.2943 & 0.5612 & 0.0880 & 0.4590 & 0.4530 \\
\hline
\end{tabular}




\begin{tabular}{|c|c|c|c|c|c|c|c|c|c|c|c|}
\hline 617 & Water & $\mathrm{CH} 3 \mathrm{OH}$ & $\mathrm{C} 2 \mathrm{H} 5 \mathrm{OH}$ & 79.33 & 10.58 & 0.1461 & 0.3496 & 0.5043 & 0.0820 & 0.5280 & 0.3900 \\
\hline 618 & Water & $\mathrm{CH} 3 \mathrm{OH}$ & $\mathrm{C} 2 \mathrm{H} 5 \mathrm{OH}$ & 84.76 & 11.30 & 0.1422 & 0.4280 & 0.4298 & 0.0730 & 0.6140 & 0.3130 \\
\hline 619 & Water & $\mathrm{CH} 3 \mathrm{OH}$ & $\mathrm{C} 2 \mathrm{H} 5 \mathrm{OH}$ & 88.84 & 11.84 & 0.1457 & 0.4946 & 0.3597 & 0.0680 & 0.6770 & 0.2550 \\
\hline 620 & Water & $\mathrm{CH} 3 \mathrm{OH}$ & $\mathrm{C} 2 \mathrm{H} 5 \mathrm{OH}$ & 97.33 & 12.98 & 0.1432 & 0.6299 & 0.2269 & 0.0570 & 0.7980 & 0.1450 \\
\hline 621 & Water & $\mathrm{CH} 3 \mathrm{OH}$ & $\mathrm{C} 2 \mathrm{H} 5 \mathrm{OH}$ & 102.23 & 13.63 & 0.1476 & 0.7032 & 0.1492 & 0.0530 & 0.8550 & 0.0920 \\
\hline 622 & Water & $\mathrm{CH} 3 \mathrm{OH}$ & $\mathrm{C} 2 \mathrm{H} 5 \mathrm{OH}$ & 64.08 & 8.54 & 0.1935 & 0.1065 & 0.7000 & 0.1440 & 0.1910 & 0.6650 \\
\hline 623 & Water & $\mathrm{CH} 3 \mathrm{OH}$ & $\mathrm{C} 2 \mathrm{H} 5 \mathrm{OH}$ & 76.25 & 10.17 & 0.1862 & 0.3062 & 0.5076 & 0.1080 & 0.4780 & 0.4140 \\
\hline 624 & Water & $\mathrm{CH} 3 \mathrm{OH}$ & $\mathrm{C} 2 \mathrm{H} 5 \mathrm{OH}$ & 87.99 & 11.73 & 0.1956 & 0.5019 & 0.3025 & 0.0880 & 0.6920 & 0.2200 \\
\hline 625 & Water & $\mathrm{CH} 3 \mathrm{OH}$ & $\mathrm{C} 2 \mathrm{H} 5 \mathrm{OH}$ & 101.27 & 13.50 & 0.1930 & 0.7015 & 0.1055 & 0.0680 & 0.8660 & 0.0660 \\
\hline 626 & Water & $\mathrm{CH} 3 \mathrm{OH}$ & $\mathrm{C} 2 \mathrm{H} 5 \mathrm{OH}$ & 66.20 & 8.83 & 0.2118 & 0.1577 & 0.6305 & 0.1470 & 0.2730 & 0.5800 \\
\hline 627 & Water & $\mathrm{CH} 3 \mathrm{OH}$ & $\mathrm{C} 2 \mathrm{H} 5 \mathrm{OH}$ & 96.35 & 12.85 & 0.2144 & 0.6332 & 0.1524 & 0.0790 & 0.8150 & 0.1060 \\
\hline 628 & Water & $\mathrm{CH} 3 \mathrm{OH}$ & $\mathrm{C} 2 \mathrm{H} 5 \mathrm{OH}$ & 64.98 & 8.66 & 0.2989 & 0.1542 & 0.5469 & 0.1910 & 0.2670 & 0.5420 \\
\hline 629 & Water & $\mathrm{CH} 3 \mathrm{OH}$ & $\mathrm{C} 2 \mathrm{H} 5 \mathrm{OH}$ & 68.29 & 9.10 & 0.2948 & 0.2044 & 0.5008 & 0.1770 & 0.3460 & 0.4770 \\
\hline 630 & Water & $\mathrm{CH} 3 \mathrm{OH}$ & $\mathrm{C} 2 \mathrm{H} 5 \mathrm{OH}$ & 73.72 & 9.83 & 0.3029 & 0.3037 & 0.3934 & 0.1600 & 0.4870 & 0.3530 \\
\hline 631 & Water & $\mathrm{CH} 3 \mathrm{OH}$ & $\mathrm{C} 2 \mathrm{H} 5 \mathrm{OH}$ & 85.84 & 11.44 & 0.2974 & 0.5007 & 0.2019 & 0.1220 & 0.7150 & 0.1630 \\
\hline 632 & Water & $\mathrm{CH} 3 \mathrm{OH}$ & $\mathrm{C} 2 \mathrm{H} 5 \mathrm{OH}$ & 89.12 & 11.88 & 0.2968 & 0.5517 & 0.1515 & 0.1150 & 0.7660 & 0.1190 \\
\hline 633 & Water & $\mathrm{CH} 3 \mathrm{OH}$ & $\mathrm{C} 2 \mathrm{H} 5 \mathrm{OH}$ & 64.82 & 8.64 & 0.3399 & 0.1536 & 0.5065 & 0.2080 & 0.2730 & 0.5190 \\
\hline 634 & Water & $\mathrm{CH} 3 \mathrm{OH}$ & $\mathrm{C} 2 \mathrm{H} 5 \mathrm{OH}$ & 85.37 & 11.38 & 0.3437 & 0.5032 & 0.1531 & 0.1340 & 0.7350 & 0.1310 \\
\hline 635 & Water & $\mathrm{CH} 3 \mathrm{OH}$ & $\mathrm{C} 2 \mathrm{H} 5 \mathrm{OH}$ & 72.39 & 9.65 & 0.3946 & 0.3055 & 0.2999 & 0.1880 & 0.5100 & 0.3020 \\
\hline 636 & Water & $\mathrm{CH} 3 \mathrm{OH}$ & $\mathrm{C} 2 \mathrm{H} 5 \mathrm{OH}$ & 80.46 & 10.73 & 0.4107 & 0.4380 & 0.1513 & 0.1630 & 0.6890 & 0.1480 \\
\hline 637 & Water & $\mathrm{CH} 3 \mathrm{OH}$ & $\mathrm{C} 2 \mathrm{H} 5 \mathrm{OH}$ & 61.84 & 8.24 & 0.4959 & 0.1529 & 0.3512 & 0.2600 & 0.2950 & 0.4450 \\
\hline 638 & Water & $\mathrm{CH} 3 \mathrm{OH}$ & $\mathrm{C} 2 \mathrm{H} 5 \mathrm{OH}$ & 64.86 & 8.65 & 0.4997 & 0.1973 & 0.3030 & 0.2480 & 0.3750 & 0.3770 \\
\hline 639 & Water & $\mathrm{CH} 3 \mathrm{OH}$ & $\mathrm{C} 2 \mathrm{H} 5 \mathrm{OH}$ & 70.66 & 9.42 & 0.4952 & 0.2988 & 0.2060 & 0.2160 & 0.5420 & 0.2420 \\
\hline 640 & Water & $\mathrm{CH} 3 \mathrm{OH}$ & $\mathrm{C} 2 \mathrm{H} 5 \mathrm{OH}$ & 73.81 & 9.84 & 0.4968 & 0.3510 & 0.1522 & 0.2030 & 0.6180 & 0.1790 \\
\hline 641 & Water & $\mathrm{CH} 3 \mathrm{OH}$ & $\mathrm{C} 2 \mathrm{H} 5 \mathrm{OH}$ & 70.42 & 9.39 & 0.5448 & 0.3064 & 0.1488 & 0.2250 & 0.5800 & 0.1950 \\
\hline 642 & Water & $\mathrm{CH} 3 \mathrm{OH}$ & $\mathrm{C} 2 \mathrm{H} 5 \mathrm{OH}$ & 59.57 & 7.94 & 0.6167 & 0.1524 & 0.2309 & 0.2940 & 0.3360 & 0.3700 \\
\hline 643 & Water & $\mathrm{CH} 3 \mathrm{OH}$ & $\mathrm{C} 2 \mathrm{H} 5 \mathrm{OH}$ & 64.32 & 8.58 & 0.6210 & 0.2272 & 0.1518 & 0.2660 & 0.4890 & 0.2450 \\
\hline
\end{tabular}




\begin{tabular}{|c|c|c|c|c|c|c|c|c|c|c|c|}
\hline 644 & Water & $\mathrm{CH} 3 \mathrm{OH}$ & $\mathrm{C} 2 \mathrm{H} 5 \mathrm{OH}$ & 56.44 & 7.52 & 0.6925 & 0.1101 & 0.1974 & 0.3310 & 0.2770 & 0.3920 \\
\hline 645 & Water & $\mathrm{CH} 3 \mathrm{OH}$ & $\mathrm{C} 2 \mathrm{H} 5 \mathrm{OH}$ & 58.71 & 7.83 & 0.6946 & 0.1540 & 0.1514 & 0.3140 & 0.3830 & 0.3030 \\
\hline 646 & Water & $\mathrm{CH} 3 \mathrm{OH}$ & $\mathrm{C} 2 \mathrm{H} 5 \mathrm{OH}$ & 61.27 & 8.17 & 0.6981 & 0.2013 & 0.1006 & 0.2970 & 0.4990 & 0.2040 \\
\hline 647 & Water & $\mathrm{CH} 3 \mathrm{OH}$ & $\mathrm{C} 2 \mathrm{H} 5 \mathrm{OH}$ & 51.46 & 6.86 & 0.7989 & 0.1021 & 0.0990 & 0.3840 & 0.3260 & 0.2900 \\
\hline 648 & Water & $\mathrm{CH} 3 \mathrm{OH}$ & & 106.01 & 14.13 & 0.2418 & 0.7582 & 0.0000 & 0.0738 & 0.9262 & 0.0000 \\
\hline 649 & Water & $\mathrm{CH} 3 \mathrm{OH}$ & & 90.50 & 12.07 & 0.4335 & 0.5665 & 0.0000 & 0.1376 & 0.8624 & 0.0000 \\
\hline 650 & Water & $\mathrm{CH} 3 \mathrm{OH}$ & & 82.05 & 10.94 & 0.5381 & 0.4619 & 0.0000 & 0.1769 & 0.8231 & 0.0000 \\
\hline 651 & Water & $\mathrm{CH} 3 \mathrm{OH}$ & & 73.84 & 9.85 & 0.6330 & 0.3670 & 0.0000 & 0.2200 & 0.7800 & 0.0000 \\
\hline 652 & Water & $\mathrm{CH} 3 \mathrm{OH}$ & & 64.23 & 8.56 & 0.7295 & 0.2705 & 0.0000 & 0.2801 & 0.7199 & 0.0000 \\
\hline 653 & Water & $\mathrm{CH} 3 \mathrm{OH}$ & & 55.38 & 7.38 & 0.8039 & 0.1961 & 0.0000 & 0.3504 & 0.6496 & 0.0000 \\
\hline 654 & Water & $\mathrm{CH} 3 \mathrm{OH}$ & & 50.97 & 6.80 & 0.8366 & 0.1634 & 0.0000 & 0.3936 & 0.6064 & 0.0000 \\
\hline 655 & Water & $\mathrm{CH} 3 \mathrm{OH}$ & & 42.88 & 5.72 & 0.8908 & 0.1092 & 0.0000 & 0.4944 & 0.5056 & 0.0000 \\
\hline 656 & Water & $\mathrm{CH} 3 \mathrm{OH}$ & & 37.99 & 5.06 & 0.9207 & 0.0793 & 0.0000 & 0.5754 & 0.4246 & 0.0000 \\
\hline 657 & Water & $\mathrm{CH} 3 \mathrm{OH}$ & & 34.57 & 4.61 & 0.9406 & 0.0594 & 0.0000 & 0.6453 & 0.3547 & 0.0000 \\
\hline 658 & Water & $\mathrm{CH} 3 \mathrm{OH}$ & & 31.95 & 4.26 & 0.9554 & 0.0446 & 0.0000 & 0.7089 & 0.2911 & 0.0000 \\
\hline 659 & Water & $\mathrm{CH} 3 \mathrm{OH}$ & & 30.08 & 4.01 & 0.9657 & 0.0343 & 0.0000 & 0.7607 & 0.2393 & 0.0000 \\
\hline 661 & Water & & $\mathrm{C} 2 \mathrm{H} 5 \mathrm{OH}$ & 58.08 & 7.74 & 0.2190 & 0.0000 & 0.7810 & 0.1839 & 0.0000 & 0.8161 \\
\hline 662 & Water & & $\mathrm{C} 2 \mathrm{H} 5 \mathrm{OH}$ & 55.71 & 7.43 & 0.4180 & 0.0000 & 0.5820 & 0.2905 & 0.0000 & 0.7095 \\
\hline 663 & Water & & $\mathrm{C} 2 \mathrm{H} 5 \mathrm{OH}$ & 53.95 & 7.19 & 0.5098 & 0.0000 & 0.4902 & 0.3210 & 0.0000 & 0.6790 \\
\hline 664 & Water & & $\mathrm{C} 2 \mathrm{H} 5 \mathrm{OH}$ & 51.17 & 6.82 & 0.6632 & 0.0000 & 0.3368 & 0.3714 & 0.0000 & 0.6286 \\
\hline 665 & Water & & $\mathrm{C} 2 \mathrm{H} 5 \mathrm{OH}$ & 50.33 & 6.71 & 0.7152 & 0.0000 & 0.2848 & 0.3895 & 0.0000 & 0.6105 \\
\hline 666 & Water & & $\mathrm{C} 2 \mathrm{H} 5 \mathrm{OH}$ & 47.21 & 6.29 & 0.7978 & 0.0000 & 0.2022 & 0.4317 & 0.0000 & 0.5683 \\
\hline 667 & Water & & $\mathrm{C} 2 \mathrm{H} 5 \mathrm{OH}$ & 45.69 & 6.09 & 0.8330 & 0.0000 & 0.1670 & 0.4551 & 0.0000 & 0.5449 \\
\hline 668 & Water & & $\mathrm{C} 2 \mathrm{H} 5 \mathrm{OH}$ & 43.42 & 5.79 & 0.8657 & 0.0000 & 0.1343 & 0.4963 & 0.0000 & 0.5037 \\
\hline 669 & Water & & $\mathrm{C} 2 \mathrm{H} 5 \mathrm{OH}$ & 38.44 & 5.12 & 0.9084 & 0.0000 & 0.0916 & 0.5666 & 0.0000 & 0.4334 \\
\hline 670 & Water & & $\mathrm{C} 2 \mathrm{H} 5 \mathrm{OH}$ & 33.17 & 4.42 & 0.9477 & 0.0000 & 0.0523 & 0.6836 & 0.0000 & 0.3164 \\
\hline 671 & & $\mathrm{CH} 3 \mathrm{OH}$ & $\mathrm{C} 2 \mathrm{H} 5 \mathrm{OH}$ & 64.24 & 8.56 & 0.0000 & 0.0841 & 0.9159 & 0.0000 & 0.1610 & 0.8390 \\
\hline
\end{tabular}




\begin{tabular}{rllllllllll}
672 & $\mathrm{CH} 3 \mathrm{OH}$ & $\mathrm{C} 2 \mathrm{H} 5 \mathrm{OH}$ & 67.85 & 9.05 & 0.0000 & 0.1353 & 0.8647 & 0.0000 & 0.2470 & 0.7530 \\
673 & $\mathrm{CH} 3 \mathrm{OH}$ & $\mathrm{C} 2 \mathrm{H} 5 \mathrm{OH}$ & 71.67 & 9.56 & 0.0000 & 0.1902 & 0.8098 & 0.0000 & 0.3310 & 0.6690 \\
674 & $\mathrm{CH} 3 \mathrm{OH}$ & $\mathrm{C} 2 \mathrm{H} 5 \mathrm{OH}$ & 78.14 & 10.42 & 0.0000 & 0.2919 & 0.7081 & 0.0000 & 0.4670 & 0.5330 \\
675 & $\mathrm{CH} 3 \mathrm{OH}$ & $\mathrm{C} 2 \mathrm{H} 5 \mathrm{OH}$ & 82.78 & 11.04 & 0.0000 & 0.3585 & 0.6415 & 0.0000 & 0.5430 & 0.4570 \\
676 & $\mathrm{CH} 3 \mathrm{OH}$ & $\mathrm{C} 2 \mathrm{H} 5 \mathrm{OH}$ & 88.24 & 11.76 & 0.0000 & 0.4414 & 0.5586 & 0.0000 & 0.6280 & 0.3720 \\
677 & $\mathrm{CH} 3 \mathrm{OH}$ & $\mathrm{C} 2 \mathrm{H} 5 \mathrm{OH}$ & 94.80 & 12.64 & 0.0000 & 0.5372 & 0.4628 & 0.0000 & 0.7150 & 0.2850 \\
678 & $\mathrm{CH} 3 \mathrm{OH}$ & $\mathrm{C} 2 \mathrm{H} 5 \mathrm{OH}$ & 101.69 & 13.56 & 0.0000 & 0.6392 & 0.3608 & 0.0000 & 0.7920 & 0.2080 \\
679 & $\mathrm{CH} 3 \mathrm{OH}$ & $\mathrm{C} 2 \mathrm{H} 5 \mathrm{OH}$ & 109.37 & 14.58 & 0.0000 & 0.7489 & 0.2511 & 0.0000 & 0.8650 & 0.1350 \\
680 & $\mathrm{CH} 3 \mathrm{OH}$ & $\mathrm{C} 2 \mathrm{H} 5 \mathrm{OH}$ & 116.14 & 15.48 & 0.0000 & 0.8535 & 0.1465 & 0.0000 & 0.9280 & 0.0720 \\
681 & $\mathrm{CH} 3 \mathrm{OH}$ & $\mathrm{C} 2 \mathrm{H} 5 \mathrm{OH}$ & 120.93 & 16.12 & 0.0000 & 0.9165 & 0.0835 & 0.0000 & 0.9610 & 0.0390 \\
\hline
\end{tabular}

BEHRENS, D. \& ECKERMANN, R. 1977-1996. Vapor-Liquid Equilibrium Data Collection, Frankfurt, DECHEMA. 